\title{
Synthesis and characterization of Locust Bean Gum derivatives and their application in the production of nanoparticles
}

Luis Braz ${ }^{\mathrm{a}, \mathrm{b}, \mathrm{c}, \mathrm{d}^{*}}$, Ana Grenha ${ }^{\mathrm{d}, \mathrm{e}}$, Marta C. Corvo ${ }^{\mathrm{f}}$, João Paulo Lourenço ${ }^{\mathrm{a}, \mathrm{g}, \mathrm{h}}$, Domingos

$5 \quad$ Ferreira $^{\mathrm{c}}$, Bruno Sarmento ${ }^{\mathrm{i}, \mathrm{j}, \mathrm{k}}$, Ana M. Rosa da Costa ${ }^{\mathrm{a}^{*}}$

${ }^{\mathrm{a} C I Q A}$ - Centre of Research in Chemistry of Algarve, Faculty of Sciences and Technology, Campus de Gambelas, 8005-139 Faro, Portugal; ${ }^{\mathrm{b}}$ Centro de Estudos e Desenvolvimento em Saúde (CESUAlg), University of Algarve, Avenida Dr. Adelino

10 da Palma Carlos, 8000-510 Faro, Portugal; ${ }^{c}$ Department of Pharmaceutical Technology, Faculty of Pharmacy, University of Porto, Rua de Jorge Viterbo Ferreira n. ${ }^{\circ}$ 228, 4050313 Porto, Portugal; ${ }^{\mathrm{d} C B M R}$ - Centre for Biomedical Research, University of Algarve, Faculty of Sciences and Technology, Campus de Gambelas, 8005-139 Faro, Portugal; ${ }^{e}$ CCMAR - Centre for Marine Sciences, University of Algarve, Campus de Gambelas, 8005-139 Faro, Portugal; fi3N|CENIMAT, Department of Materials Science, Faculty of Science and Technology, UNL, 2829-516 Caparica, Portugal; ' ${ }^{\text {Department }}$ of Chemistry and Pharmacy, Faculty of Science and Technology, University of Algarve, Campus de Gambelas, 8005-139 Faro, Portugal; ${ }^{\mathrm{h}} \mathrm{CQE}-$ Centro de Química Estrutural, Instituto Superior Técnico, University of Lisbon; i i3S, Instituto de Investigação e Inovação em

20 Saúde, Universidade do Porto, Rua Alfredo Allen, 208, 4200-135 Porto, Portugal; jINEB, Instituto de Engenharia Biomédica, Biocarrier Group, Universidade do Porto, Rua Alfredo Allen, 208, 4200-135 Porto, Portugal; ' Instituto de Investigação e Formação Avançada em Ciências e Tecnologias da Saúde, CESPU, Rua Central de Gandra, 1317, 4585-116 Gandra, Portugal 
*Corresponding author:

Luis Braz

School of Health - University of Algarve

Avenida Dr. Adelino da Palma Carlos

$30 \quad 8000-510$ Faro, Portugal

Tel.: +351 289800100 - Ext. 7835

Fax: +351289818419

E-mail address: lvbraz@ualg.pt

35 Ana Maria Rosa da Costa

CIQA - Centre of Research in Chemistry of Algarve

Faculty of Sciences and Technology - University of Algarve

Campus de Gambelas

8005-139 Faro

$40 \quad$ Portugal

Tel.: +351289800100

Fax: +351289800066

E-mail address: amcosta@ualg.pt 
45 Abstract (150)

The development of LBG-based nanoparticles intending an application in oral immunization is presented. Nanoparticle production occurred by mild polyelectrolyte complexation, requiring the chemical modification of LBG. Three LBG derivatives were synthesized, namely a positively charged ammonium derivative (LBGA) and 50 negatively charged sulfate (LBGS) and carboxylate (LBGC) derivatives. These were characterized by Fourier-transform infrared spectroscopy, elemental analysis, nuclear magnetic resonance spectroscopy, gel permeation chromatography, and x-ray diffraction. As a pharmaceutical application was aimed, a toxicological analysis of the derivatives was performed by both MTT test and LDH release assay.

55 Several nanoparticle formulations were produced using LBGA or chitosan (CS) as positively charged polymers, and LBGC or LBGS as negatively charged counterparts, producing nanoparticles with adequate properties regarding an application in oral immunization.

60 Highlights 3 to 5 bullet points (max 85 characters each, including spaces)

- LBG charged derivatives (ammonium, sulfated and carboxylated) were synthesized;

- LBG-based nanoparticles, adequate for drug delivery applications, were produced;

- Only the LBG ammonium derivative demonstrated severe cytotoxicity in Caco-2 cells;

- Nanoparticles evidenced very mild effect on Caco-2 cell viability. 
Keywords: Carboxylated locust bean gum; Polyelectrolyte complexation; Polymeric nanoparticles; Sulfated locust bean gum; Tetraalkylammonium locust bean gum

\section{Introduction}

The recent decades have brought to the market many new biomolecules that have been identified as having therapeutic potential. These molecules, which include from proteins and peptides to antigens and nucleic acids, are usually called biopharmaceuticals, meaning that they are biological in nature and manufactured using biotechnology (Rader, 2008). Although therapeutically promising, biopharmaceuticals are very unstable compounds and their administration is extremely challenging, due to inherent physicochemical and biopharmaceutical properties (Alonso, 2004; Kammona \&

80 Kiparissides, 2012). Moreover, the therapeutic action of proteins and protein-based molecules is not only limited by the potential degradation in biological environments, but also compromised by their low ability to reach the therapeutic site of action (Antosova, Mackova, Kral \& Macek, 2009; Casettari \& Illum, 2014; Kammona \& Kiparissides, 2012). As such, a meaningful challenge for current pharmaceutical scientists has been the need to develop suitable vehicles that permit delivering macromolecules through alternative routes of administration. Polymeric nanoparticles have been demonstrating to be very promising in oral delivery of biopharmaceuticals, as many works report their effective role in the enhancement of oral drug bioavailability by facilitating cell internalization (Csaba, Garcia-Fuentes \& Alonso, 2006; Kadiyala,

90 Loo, Roy, Rice \& Leong, 2010). Their reduced size provides an intimate contact with epithelia and, in several occasions, they have shown the capacity to carry the encapsulated molecules through the epithelium (Csaba, Garcia-Fuentes \& Alonso, 2006; de la Fuente, Csaba, Garcia-Fuentes \& Alonso, 2008). With respect to oral vaccination, 
the design of suitable antigen delivery systems should focus on optimizing antigen association efficiency, ensuring the maintenance of its stability during association, tailoring release kinetics and eliciting high levels of long-lasting antibody and cellular immune responses. Nanoparticles may provide extra benefits in oral immunization strategies, because Peyer's Patches (PPs) have shown to be a predominant site for uptake of particulates (Lavelle \& O'Hagan, 2006). Given their role in intestinal mediated immunization, $\mathrm{M}$ cells are the primary targets to consider. A careful selection of nanoparticle matrix materials may further help on the potentiation of an immune response. In this context, mucoadhesive polymers can contribute to the prolongation on the intestinal residence time (Arca, Gunbeyaz \& Senel, 2009), potentiating the uptake by $\mathrm{M}$ cells.

105 Locust bean gum (LBG), also known as carob bean gum, is obtained from the endosperm of carob tree (Ceratonia siliqua) seeds, where it acts as reserve material. It is reported as biocompatible, biosorbable, biodegradable, non-teratogenic and nonmutagenic, presenting a mucoadhesive behaviour, and its degradation products are excreted readily (Dionísio \& Grenha, 2012; Malik, Arora \& Singh, 2011a; Pollard et al.,

110 2007; Sudhakar, Kuotsu \& Bandyopadhyay, 2006; Surana, Munday, Cox \& Khan, 1998). Classified by the FDA as a GRAS (Generally Recognized as Safe) material, it is approved in most areas of the world for use in the food industry as thickener, stabilizer, emulsifier, and gelling agent (E410). It is also used in the pharmaceutical industry as excipient in drug formulations, and in biomedical applications (Barak \& Mudgil, 2014;

115 Dionísio \& Grenha, 2012; Kawamura, 2008; Prajapati, Jani, Moradiya, Randeria \& Nagar, 2013). LBG is mainly comprised of high molecular weight (approximately 50 000 - $3000000 \mathrm{Da}$ ) neutral galactomannan, consisting in a linear chain of (1-4)-linked $\beta$-D-mannopyranosyl units with (1-6)-linked $\alpha$-D-galactopyranosyl residues as side 
chains. The mannose and galactose contents have been reported to be $73-86 \%$ and $27-$

$12014 \%$, respectively, which corresponds to a mannose:galactose (M/G) ratio of approximately 4:1 (Kawamura, 2008).

Recently, there has been a growing interest in the chemical functionalization of polysaccharides, particularly those non-animal derived, mainly by making use of the free hydroxyl groups distributed along their backbone, in order to create derivatives

125 with properties tailored for the desired applications (Mizrahy \& Peer, 2012). In this paper, the chemical modification of LBG, aimed at obtain charged derivatives intended for the development of nanoparticulate carriers by polyelectrolyte complexation, is described. Two anionic (sulfate - LBGS and carboxylate - LBGC) and one cationic (trimethylammonium - LBGA) derivatives were prepared (Figure 1). The

130 former were combined with the positively charged polysaccharide chitosan (CS) and the latter with LBGS in order to produce polymeric nanoparticles. 


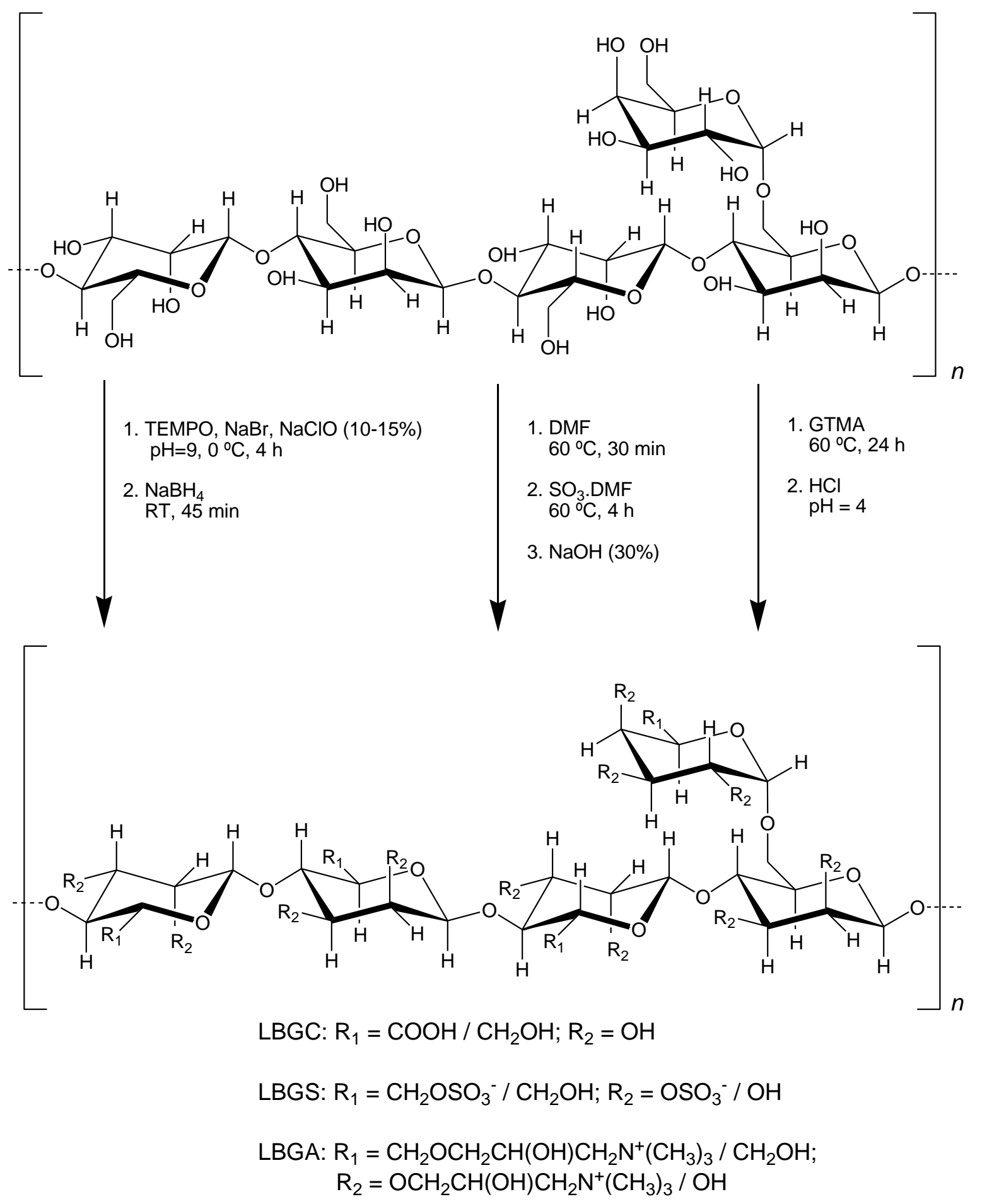

Figure 1 - Scheme of the chemical modifications introduced in LBG.

2. Materials and methods

\subsection{Materials}

Locust bean gum (LBG) was a kind gift from Industrial Farense (Faro, Portugal). Due to the presence of around 3-7\% protein in commercial LBG (Kawamura, 2008), a 
purification step, based on previously published protocols (Bouzouita et al., 2007;

140 Wang, Wang \& Sun, 2002), was performed prior to use. This purified LBG was the material used for subsequent work, unless stated otherwise. Chitosan (CS, low molecular weight, deacetylation degree $=75-85 \%$ ), glacial acetic acid, chlorosulfuric acid $\left(\mathrm{HClSO}_{3}\right.$ ), dimethylformamide (DMF), $N$-glycidyl- $N, N, N$-trimethylammonium chloride (GTMAC), sodium hydroxide $(\mathrm{NaOH})$, potassium hydroxide $(\mathrm{KOH})$, 2,2,6,6tetramethypiperidine-1-oxyl (TEMPO), sodium bromide ( $\mathrm{NaBr}$ ), sodium hypochlorite solution $(\mathrm{NaClO})$, sodium borohydride $\left(\mathrm{NaBH}_{4}\right)$, sodium nitrate $\left(\mathrm{NaNO}_{3}\right)$, sodium dihydrogen phosphate $\left(\mathrm{NaH}_{2} \mathrm{PO}_{4}\right)$, sodium azide $\left(\mathrm{NaN}_{3}\right)$, dialysis tubing (pore size $2000 \mathrm{Da}$ ), phosphotungstate dibasic hydrate, glycerol, phosphate buffered saline (PBS) pH 7.4 tablets, Dulbecco’s modified Eagle's medium (DMEM), penicillin/streptomycin

150 (10000 units/mL, $10000 \mu \mathrm{g} / \mathrm{mL}$ ), non-essential amino acids, L-glutamine $200 \mathrm{mM}$, trypsin-EDTA solution (2.5 g/L trypsin, $0.5 \mathrm{~g} / \mathrm{L}$ EDTA), trypan blue solution (0.4\%), thiazolyl blue tetrazolium bromide (MTT), lactate dehydrogenase (LDH) kit, sodium dodecyl sulfate (SDS), dimethyl sulfoxide (DMSO), hydrochloric acid ( $\mathrm{HCl} 37 \%$ ), sodium chloride $(\mathrm{NaCl})$ and potassium dihydrogen phosphate $\left(\mathrm{KH}_{2} \mathrm{PO}_{4}\right)$ were purchased from Sigma-Aldrich (Germany). Ethanol was supplied by VWR. Potassium bromide (KBr) was obtained from Riedel-del-Haën (Germany). Fetal bovine serum (FBS) was obtained from Gibco (USA). Ultrapure water (Mili-Q Plus, Milipore Iberica, Madrid, Spain) was used throughout. All other chemicals were reagent grade.

\section{$160 \quad$ 2.2. Cell line}

The Caco-2 cell line was obtained from the American Type Culture Collection (Rockville, USA) and used between passages 77-93. Cell cultures were grown in $75 \mathrm{~cm}^{2}$ flasks in humidified $5 \% \mathrm{CO}_{2} / 95 \%$ atmospheric air incubator at $37{ }^{\circ} \mathrm{C}$. Cell culture 
medium was DMEM supplemented with $10 \%$ (v/v) FBS, $1 \%$ (v/v) L-glutamine

penicillin/streptomycin. Medium was changed every 2-3 days and cells were subcultured weekly.

\subsection{Synthesis of Locust Bean Gum derivatives}

\subsubsection{Purification of Locust Bean Gum}

LBG purification was performed as described elsewhere (Braz, Grenha, Ferreira, Rosa da Costa, Gamazo \& Sarmento, 2017).

\subsubsection{Sulfation of Locust Bean Gum}

175 The sulfation agent, $\mathrm{SO}_{3} \cdot \mathrm{DMF}$, was prepared as described before (Braz, Grenha, Ferreira, Rosa da Costa, Gamazo \& Sarmento, 2017).

\section{Method 1}

LBG (500 mg) was dispersed in DMF (35 mL) and stirred at $60^{\circ} \mathrm{C}$ for $30 \mathrm{~min}$, in order

180 to provide the dispersion of LBG in the solvent. Then, the $\mathrm{SO}_{3}$ DMF complex was added $(9.3 \mathrm{~mL})$ and the mixture reacted for $4 \mathrm{~h}$ under magnetic stirring. Subsequently, the mixture was cooled down to room temperature in an ice bath, neutralized with $30 \%$ $\mathrm{NaOH}$ solution until precipitation, and concentrated under reduced pressure at $60^{\circ} \mathrm{C}$ to evaporate the solvent. The residue was dissolved in distilled water $(30 \mathrm{~mL})$ and dialyzed

185 against distilled water ( $5 \mathrm{~L}$ ). The water was changed every $24 \mathrm{~h}$ and, after 3 days, the solution was concentrated under reduced pressure at $40^{\circ} \mathrm{C}$. Then, ethanol was added to the concentrated solution, in order to precipitate the solute, and the dispersion was concentrated under reduced pressure at $40{ }^{\circ} \mathrm{C}$. The previous step was repeated twice, 
and the last evaporation was performed until full evaporation of the solvent. The obtained powder was dried in a vacuum oven at $40^{\circ} \mathrm{C}$ for 3 days, affording $407 \mathrm{mg}$ of brownish powder that was grinded and stored until further use.

\section{Method 2}

LBG sulfation by this method was performed as described before (Braz, Grenha,

195 Ferreira, Rosa da Costa, Gamazo \& Sarmento, 2017).

\subsubsection{Carboxylation of Locust Bean Gum}

LBG (500 mg) was dissolved in $200 \mathrm{~mL}$ of distilled water under stirring at $80{ }^{\circ} \mathrm{C}$ for 30 min. After cooling down, the volume was adjusted to $200 \mathrm{~mL}$ and the solution was cooled in an ice bath. Then, TEMPO (10 mg) and $\mathrm{NaBr}(50 \mathrm{mg})$ were added to the solution under stirring. A $15 \%$ sodium hypochlorite solution $(3.0 \mathrm{~mL})$ with $\mathrm{pH}$ adjusted to 9.3 with $2 \mathrm{M} \mathrm{HCl}$ solution, was mixed with the polymer solution. The $\mathrm{pH}$ was maintained at 9.3 by addition of a $0.05 \mathrm{M}$ aqueous $\mathrm{NaOH}$ solution for $4 \mathrm{~h}$. To stop the reaction, sodium borohydride $(75 \mathrm{mg})$ was added and the solution was stirred for 45 min. Then the $\mathrm{pH}$ of the mixture was adjusted to 8 by addition of $\mathrm{HCl}$ before precipitation by 2 volumes of ethanol in presence of $\mathrm{NaCl}$ (up to $10 \mathrm{~g} / \mathrm{L}$ ). The polymer was isolated by filtration under reduced pressure, washed several times with ethanol, filtered and dried in a vacuum oven at $30^{\circ} \mathrm{C}$ during 3 days. A white powder $(529 \mathrm{mg})$ was obtained, grinded and stored until further use.

\subsubsection{Quaternary ammonium salt of Locust Bean Gum}

An aqueous solution $(10 \mathrm{~mL})$ of $\mathrm{KOH}(0.550 \mathrm{~g})$, was prepared in a round bottom flask, under stirring, at $60^{\circ} \mathrm{C}$. Then, purified LBG (506 mg) and $3.72 \mathrm{~mL}$ of GTMAC were 
added. After $5 \mathrm{~h}$, an equal amount of GTMAC was added to the mixture, which was

215 allowed to react until the completion of $24 \mathrm{~h}$. It was then diluted with $20 \mathrm{~mL}$ of miliQ water, allowed to cool down to room temperature, and neutralized with $\mathrm{HCl}(2 \mathrm{M})$. The resulting solution was dialyzed for 3 days, the water being replaced every $24 \mathrm{~h}$. Then, the LBGA solution was concentrated under reduced pressure at $40^{\circ} \mathrm{C}$ and ethanol was added to the concentrated solution, in order to precipitate the solute. The dispersion was concentrated under reduced pressure at $40{ }^{\circ} \mathrm{C}$ and ethanol was added again and evaporated under the same conditions until full evaporation of the solvent. The obtained powder was dried in a vacuum oven at $40{ }^{\circ} \mathrm{C}$ for 3 days, affording $423 \mathrm{mg}$ of white powder that was grinded and stored until further use.

\subsection{Chemical characterization of Locust Bean Gum derivatives}

\subsubsection{Fourier transform infrared (FTIR) spectroscopy}

For recording FTIR spectra of purified LBG and their derivatives, samples were grounded with $\mathrm{KBr}$ in a mortar and compressed into discs. For each spectrum, a 32-scan interferogram was collected in transmittance mode with a $4 \mathrm{~cm}^{-1}$ resolution in the 4,000 $230400 \mathrm{~cm}^{-1}$ region.

\subsubsection{Elemental analysis}

Elemental analysis data were obtained in a Thermo Finnigan, FLASH EA 1112 Series

$(\mathrm{C}, \mathrm{N}, \mathrm{S})$ or in a Fisons Instruments, EA 1108 CHNS-O (O) elemental analyzer.

\subsubsection{Nuclear magnetic resonance (NMR) spectroscopy}

All liquid NMR spectra were acquired in a Bruker Avance III 400 spectrometer equipped with a temperature control unit and a pulse gradient unit capable of producing 
magnetic field pulsed gradients in the z-direction of $56.0 \mathrm{G} / \mathrm{cm}$, operating at 400.15

$240 \mathrm{MHz}$ for hydrogen, $100.61 \mathrm{MHz}$ for carbon, using a multinuclear reverse $5 \mathrm{~mm}$ probe (TXI). The samples where dissolved in $\mathrm{D}_{2} \mathrm{O}$. Solid state NMR spectra were acquired in a Bruker Avance III 300 spectrometer equipped with a BBO probehead, operating at 300.15 MHz for hydrogen, $75.00 \mathrm{MHz}$ for carbon. The sample was spun at the magic angle at a frequency of $5 \mathrm{kHz}$ in a $4 \mathrm{~mm}$-diameter rotor at room temperature.

$245 \quad{ }^{1} \mathrm{H}$ NMR spectra were recorded with $8.22 \mathrm{KHz}$ spectral window digitized with $64 \mathrm{~K}$ points. The ${ }^{13} \mathbf{C}$ spectra were recorded between 0 and 238 ppm using 24,000 Hz spectral window digitized into $64 \mathrm{~K}$ points.

Two-dimensional ${ }^{1} \mathrm{H}^{-1} \mathrm{H}$ correlation spectroscopy (COSY) spectra were acquired using 32 transients and 16 dummy scans, with a spectral width of 5000 in a total of $2 \mathrm{~K}$ data 250 points in $F 2$ and 128 data points in $F 1$, the relaxation delay was set to $1.5 \mathrm{~s}$. Heteronuclear Single Quantum Coherence-Total Correlation Spectroscopy $\left({ }^{1} \mathbf{H} /{ }^{\mathbf{1 3}} \mathbf{C}\right.$ HSQC-TOCSY) spectra were acquired using the following parameters: $2 \mathrm{~K}$ data points in F2 with a spectral width of $5000 \mathrm{~Hz}, 512$ data points in F1 with a spectral width of 17 $\mathrm{KHz}$, a relaxation delay of $2 \mathrm{~s}, \mathrm{MLEV}-17$ sequence with a mixing time of $40 \mathrm{~ms}, 16$ transients and 16 dummy scans. The phase-edited heteronuclear single quantum correlation $\left({ }^{1} \mathbf{H} /{ }^{13} \mathbf{C}\right.$ HSQC-DEPT $)$ spectra were acquired in $2 \mathrm{~K}$ data points in $\mathrm{F} 2$ with a spectral width of $5000 \mathrm{~Hz}, 512$ data points in F1 with a spectral width of $17 \mathrm{KHz}$, a relaxation delay of 2 s, 2 to 8 transients and 16 dummy scans. The Heteronuclear Multiple Bond Correlation $\left({ }^{1} \mathbf{H} /{ }^{13} \mathbf{C} \mathbf{H M B C}\right)$ spectra were acquired using the following parameters: $1 \mathrm{~K}$ data points in F2 with a spectral width of $5000 \mathrm{~Hz}, 256$ data points in F1 with a spectral width of $22 \mathrm{KHz}$, a relaxation delay of $2 \mathrm{~s}, 24$ transients and 16 dummy scans. 
The ${ }^{13} \mathrm{C}$ MAS NMR experiments were acquired with proton cross polarization (CPMAS) with a contact time of $1.2 \mathrm{~ms}$, and a recycle delay of $2.0 \mathrm{~s}$.

\subsubsection{GPC/SEC ${ }^{3}$ analysis}

Triple detection Gel Permeation Chromatography $\left(\mathrm{GPC} / \mathrm{SEC}^{3}\right)$ analysis was performed in a modular system constituted by a degasser, HPLC pump (K-1001) and RI detector (K-2300) from Knauer, and a viscometer and RALLS from Viscotek (Trisec Dual Detector Model 270), using two PL aquagel-OH mixed $8 \mu \mathrm{m}, 300$ x 7.5 mm columns. For purified LBG, LBGC and LBGS the eluent was $0.2 \mathrm{M} \mathrm{NaNO}_{3}, 0.01 \mathrm{M} \mathrm{NaH}_{2} \mathrm{PO}_{4}$, $0.1 \% \mathrm{w} / \mathrm{v} \mathrm{NaN}_{3}, \mathrm{pH}=7$, at $1 \mathrm{~mL} / \mathrm{min}$; the samples were dissolved in the eluent at 1 $\mathrm{mg} / \mathrm{mL}$. For LBGA the eluent was $0.5 \mathrm{M} \mathrm{NaNO}_{3}, 0.01 \mathrm{M} \mathrm{KH}_{2} \mathrm{PO}_{4}, 0.1 \%$ w/v NaN , $\mathrm{pH}=2$, at the same rate; the sample was dissolved at $1 \mathrm{mg} / \mathrm{mL}$ in $10^{-2} \mathrm{M} \mathrm{HCl}$.

\subsubsection{X-ray diffraction (XRD)}

Powder X-ray diffractograms were recorded on a Panalytical X'Pert Pro diffractometer, operating at $45 \mathrm{kV}$ and $35 \mathrm{~mA}$. The patterns of the pristine and modified samples were recorded in the range 5-45 degrees $(2 \theta)$ with a step size of $0.0167^{\circ}$ and a time per step of 2000 seconds, using $\mathrm{CuK} \alpha$ radiation filtered by $\mathrm{Ni}$ and an $\mathrm{X}^{\prime}$ Celerator detector. Prior to the analysis, samples were reduced to a fine powder by grinding in a mortar.

\subsection{Production, characterization and safety evaluation of Locust Bean Gum-based nanoparticles}

285 All nanoparticles were prepared by polyelectrolyte complexation, which consists in the electrostatic interaction between the positive and negative charges of the different polymers (Bhattarai, Gunn \& Zhang, 2010). 


\subsubsection{Production of CS/LBGS and CS/LBGC nanoparticles}

290 Several mass ratios of CS/LBGC and CS/LBGS (see Table 1) were used to prepare the nanoparticles by polyelectrolyte complexation. The stock solution of CS, dissolved in $1 \%(\mathrm{w} / \mathrm{w})$ acetic acid, was prepared to reach a final concentration of $1.0 \mathrm{mg} / \mathrm{mL}$, while those of LBGC and LBGS, dissolved in ultrapure water, had a final concentration of 2.0 mg/mL. Nanoparticles were prepared as described before (Braz, Grenha, Ferreira, Rosa 295 da Costa, Gamazo \& Sarmento, 2017).

\subsubsection{Production of LBGA/LBGS nanoparticles}

Three mass ratios of LBGA/LBGS (2/1, 1/1 and 1/2) were used to prepare the nanoparticles by polyelectrolyte complexation. The stock solutions of LBGA and LBGS were prepared by dissolving the polymers in ultrapure water, at final concentrations of $0.5 \mathrm{mg} / \mathrm{mL}$ and $1.0 \mathrm{mg} / \mathrm{mL}$, respectively. Nanoparticles were prepared as described before (Braz, Grenha, Ferreira, Rosa da Costa, Gamazo \& Sarmento, 2017).

\subsubsection{Characterization of Locust Bean Gum-based nanoparticles}

\subsubsection{Size, polydispersion index, $\zeta$ potential and production yield}

The size, $\zeta$ potential, polydispersion index $(\mathrm{PdI})$ and production yield were determined as described before (Braz, Grenha, Ferreira, Rosa da Costa, Gamazo \& Sarmento, 2017).

\subsubsection{Morphological analysis}

The morphological examination of LBGA/LBGS nanoparticles was conducted by transmission electron microscopy (TEM; JEM-1011, JEOL, Japan). The samples were 
stained with $2 \%(\mathrm{w} / \mathrm{v})$ phosphotungstic acid and placed on copper grids with carbon films (Ted Pella, USA) for TEM observation.

\subsubsection{Safety evaluation}

The in vitro cell viability and cytotoxicity of bulk LBG, purified LBG and the synthesized derivatives was assessed in Caco-2 cells by the MTT and the LDH release assays, respectively. LBGA/LBGS nanoparticles were evaluated using the MTT assay. Gamazo \& Sarmento, 2017).

\subsection{Statistical analyses}

The t-test and the one-way analysis of variance (ANOVA) with the pair wise multiple comparison procedures (Holm-Sidak method) were performed to compare two or multiple groups, respectively. All analyses were run using the SigmaStat statistical program (Version 3.5, SyStat, USA) and differences were considered to be significant at a level of $P<0.05$.

\section{Results and discussion}

\subsection{Synthesis and chemical characterization of Locust Bean Gum derivatives}

The syntheses of the three charged LBG derivatives were made by adapting procedures described in the literature for the modification of other polysaccharides. To perform the sulfation reaction, $\mathrm{SO}_{3}$.DMF was chosen as sulfating agent (Yuan et al., 2005), as it presents advantages over methods involving the manipulation of either pyridine or sulfur trioxide (Alban, Schauerte \& Franz, 2002; Mähner, Lechner \& Nordmeier, 2001; Mihai, Mocanu \& Carpov, 2001). For the synthesis of the sulfate derivative, two 
approaches were performed as described in the methodology. The difference mainly resided in the processing of $\mathrm{LBG}$ prior to the addition of $\mathrm{SO}_{3} \cdot \mathrm{DMF}$. For the introduction

340 of trimethylammonium groups in LBG, GTMAC was used as alkylating agent, which proved to be efficient in the alkylation of other polysaccharides (Dionísio, Braz, Corvo, Lourenço, Grenha \& da Costa, 2016; Qin et al., 2004; Rekha \& Sharma, 2009;

Simkovic, Yadav, Zalibera \& Hicks, 2009).

For the transformation of LBG into the corresponding polyuronic acid, TEMPO, a

345 stable nitroxyl radical, was chosen as oxidizing agent (Sierakowski, Milas, Desbrières $\&$ Rinaudo, 2000). This has proved to possess a high efficiency in the conversion of high molecular weight polysaccharides. A highly selective oxidation of C-6 primary hydroxyl to carboxylic groups can be achieved in an aqueous solution of the polysaccharide at $\mathrm{pH}$ 9-11 with $\mathrm{NaClO}$ and catalytic amounts of TEMPO and $\mathrm{NaBr}$

350 (Cunha, Maciel, Sierakowski, Paula \& Feitosa, 2007; da Silva Perez, Montanari \& Vignon, 2003; Sierakowski, Milas, Desbrières \& Rinaudo, 2000).

As shown in Figure 2, LBG sulfate functionalization (LBGS) was confirmed by FTIR, through the appearance of a $\mathrm{S}=\mathrm{O}$ asymmetric stretching band (Yuan et al., 2005) at $1255 \mathrm{~cm}^{-1}$ and that of C-O-S symmetric stretching (Alban, Schauerte \& Franz, 2002) at

$355817 \mathrm{~cm}^{-1}$. In the carboxylate derivative (LBGC), the absorption bands at $1601 \mathrm{~cm}^{-1}$ and $1415 \mathrm{~cm}^{-1}$ are attributed to asymmetric and symmetric stretching vibration of $-\mathrm{COO}^{-}$, respectively (Cunha, Maciel, Sierakowski, Paula \& Feitosa, 2007). Since the quaternary ammonium groups do not display characteristic IR absorption bands (Nakanishi, Goto \& Ohashi, 1957), evidence for formation of the amino functionalized derivative

360 (LBGA) comes from the broadening of the band at $1088 \mathrm{~cm}^{-1}$ (ether C-O symmetric stretching) and the new bands at 1479 and $914 \mathrm{~cm}^{-1}$ (C-H scissoring in methyl groups of the ammonium and ether C-O asymmetric stretching, respectively) (Qin et al., 2004). 

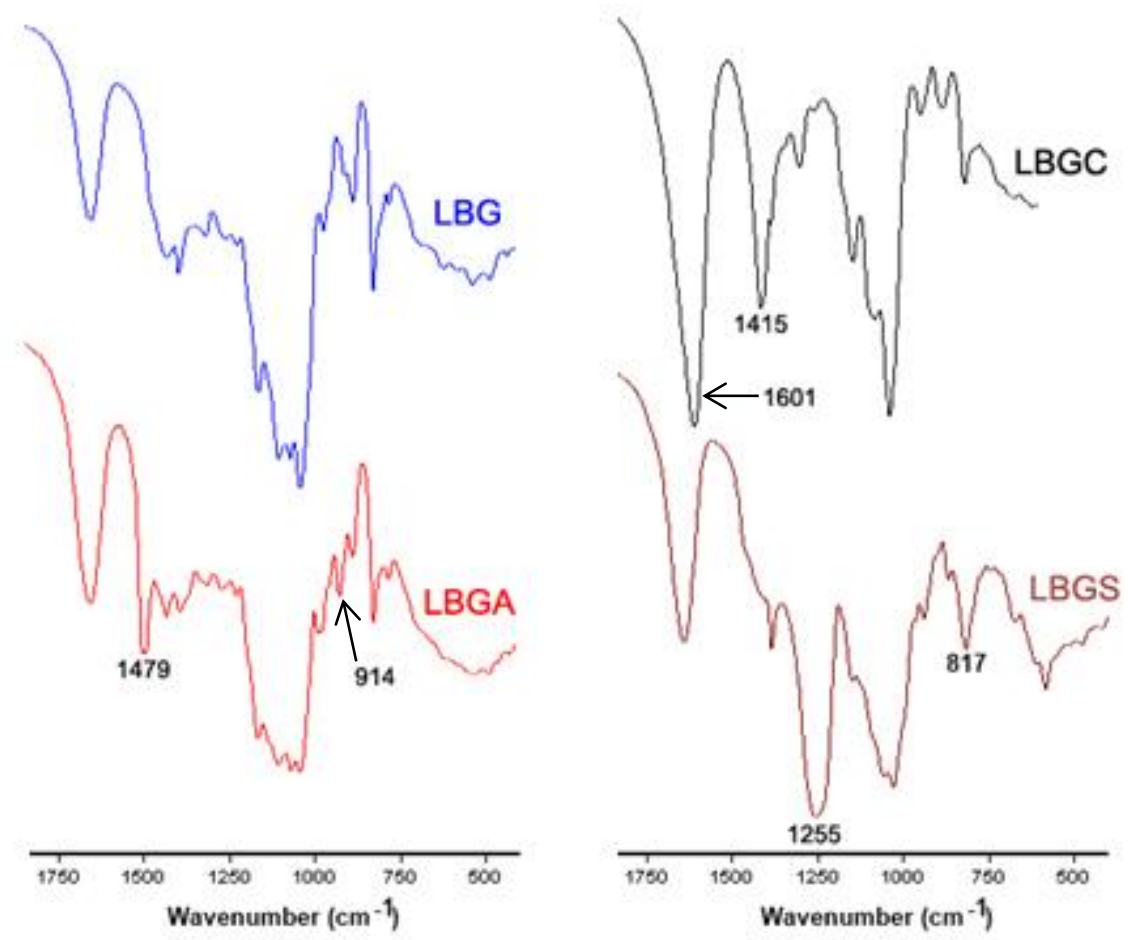

365 Figure 2 - FTIR spectra of purified Locust Bean Gum (LBG) and its ammonium (LBGA), carboxylate (LBGC) and sulfate (LBGS-M1) derivatives.

In the elemental analysis, the weight percentages found for the analysed elements are compiled in Table S1.

370 For LBGS, different degrees of substitution were obtained, even under the same reaction conditions. For the sample of LBGS obtained by method 1 (LBGS-M1), a C:S molar ratio of 8.78 was obtained, which corresponds to a degree of substitution (DS) of 3.5. Therefore, if sulfate groups are assumed to be in the form of sodium salts, a molecular formula between $\mathrm{C}_{30} \mathrm{H}_{47} \mathrm{~S}_{3} \mathrm{O}_{34} \mathrm{Na}_{3}$ and $\mathrm{C}_{30} \mathrm{H}_{46} \mathrm{~S}_{4} \mathrm{O}_{37} \mathrm{Na}_{4}$, to which corresponds 375 a mean molecular weight of $1166 \mathrm{~g} / \mathrm{mol}$, is derived. On the other hand, the samples of LBGS obtained by method 2 presented a high variability on C:S molar ratio, ranging from 26.76 in batch 1 (LBGS-M2-B1) to 6.55 in batch 2 (LBGS-M2-B2), and batch 3 (LBGS-M2-B3) presenting a value of 10.24. These values corresponded to values of DS of $1.22,4.63$, and 3 , and to the mean molecular weights of 932,1282 , and $1111 \mathrm{~g} / \mathrm{mol}$, 
respectively. As indicated in materials and methods, and stated above, the difference between the two methods only refers to a preliminary treatment of LBG before the sulfation reaction. In the second method, a better dispersion of LBG was promoted before the contact with the sulfating agent in an attempt to improve the reaction. The need for this pre-treatment was motivated by the poor solubility of LBG in DMF. Since 385 in the sulfation reaction the polymer is used as a dispersion in the solvent, it would be expected that a more effective dispersion would favour the reaction. Quite surprisingly, it was observed that, although the pre-treatment afforded the highest value of DS (4.63), it also gave the lowest substitution (1.22), while in its absence an intermediate value of DS was obtained. This variation in DS translates, in the FTIR spectra of the various 390 samples, in different intensities of the band at $1255 \mathrm{~cm}^{-1}$ relative to other bands in the spectrum, with more substituted samples presenting a more intense band (Figure S1). Assuming that better dispersion of LBG leads to higher reaction efficiency and affords higher values of DS, it seems that the dispersibility of LBG in the reaction medium does not directly correlate to the method used in its dispersion. One reason for the observed 395 variability in DS may be the fact that, contrary to what is observed in the reactions described below (oxidation and alkylation), in which LBG progressively dissolves as the reactions proceed, in this case a total solubilisation is never reached. This renders the outcome of this reaction quite unpredictable and, therefore, this issue will have to be tackled in future work. In fact, the reaction of LBG activated by pre-soaking in DMF 400 and dispersed in the same solvent, with solid $\mathrm{SO}_{3}$. DMF complex, bellow $15^{\circ} \mathrm{C}$, led to a DS of approximately 4 (Maiti, Chowdhury, Chakraborty, Ray \& Sa, 2014). On the other hand, sulfation of LBG dispersed in formamide with $\mathrm{SO}_{3}$.pyridine complex, under diverse conditions of reaction time, temperature, and amount of sulfating agent, led to DS varying between approximately 2 and nearly 5 (Wang et al., 2014). Again, the 
soaking of LBG with the solvent prior to the reaction led to an intermediate DS relative to the range obtained without any pre-treatment, although in the latter case a different reagent and solvent were used. Nevertheless, only one batch per reaction conditions seems to have been obtained in both these works and, therefore, the state of dispersion of LBG in each case may well be the factor governing the substitution obtained, instead 410 of the parameters analysed. Moreover, in the latter work, no correlation or trend between molecular weights of the obtained derivatives or depolymerization of the parent polysaccharide and degree of substitution is observed. On the contrary, a very erratic dispersion of molecular weights with growing DS is obtained, pointing to a random behaviour in this reaction.

415 For LBGC, a C:O ratio of 1.02 was found, which corresponds to a degree of oxidation (DO) of 4, meaning that all the free C-6 must have been oxidized. Assuming all carboxylate groups to be in the sodium salt form, the molecular formula would be $\mathrm{C}_{30} \mathrm{H}_{38} \mathrm{O}_{29} \mathrm{Na}_{4}$, and the molecular weight $955 \mathrm{~g} / \mathrm{mol}$. This value is not surprising, in view of the effectiveness of the oxidizing system, although somewhat higher than DO 420 values observed for other galactomannans, which typically lay below $70 \%$ of the free units (Cunha, Maciel, Sierakowski, Paula \& Feitosa, 2007).

In LBGA, the C:N molar ratio was found to be 13.16 , corresponding to a DS of 4.24. If all the ammonium groups are in the form of chloride salt, this corresponds to a molecular formula between $\mathrm{C}_{54} \mathrm{H}_{106} \mathrm{O}_{29} \mathrm{~N}_{4} \mathrm{Cl}_{4}$ and $\mathrm{C}_{60} \mathrm{H}_{120} \mathrm{O}_{30} \mathrm{~N}_{5} \mathrm{Cl}_{5}$, and the mean 425 molecular weight of $1454 \mathrm{~g} / \mathrm{mol}$. This corresponds to a full reaction of the free C-6 hydroxyl groups, along with reaction on some secondary hydroxyls, in line with what was observed by us in a similar modification performed in pullulan (Dionísio, Braz, Corvo, Lourenço, Grenha \& da Costa). 
The analysis of the ${ }^{1} \mathrm{H}$ NMR and ${ }^{13} \mathrm{C}$ spectra of LBG and the obtained derivatives

430 (Figure S2) allowed us to obtain a molecular view on the success of the transformations. However, the broadened signals in the ${ }^{1} \mathrm{H}$ spectra do not allow the evaluation of the derivatization locations and, therefore, spectral assignment was performed through 2D NMR $\left({ }^{1} \mathrm{H},{ }^{1} \mathrm{H}-\mathrm{COSY}\right.$ and $\left.{ }^{13} \mathrm{C},{ }^{1} \mathrm{H}-\mathrm{HSQC}-\mathrm{DEPT}\right)$ experiments. Figure 3 shows the ${ }^{1} \mathrm{H} /{ }^{13} \mathrm{C}$ HSQC-DEPT NMR spectra of untreated LBG (a), LBGS-

435 M2-B1 (b), LBGC (c), and LBGA (d). In untreated LBG, the two anomeric carbons C-1 from mannose and galactose residues resonate at 102.8 and $100.3 \mathrm{ppm}$, respectively. The unsubstituted C- 6 positions of main chain mannose exhibited a chemical shift at $61.4 \mathrm{ppm}$. 


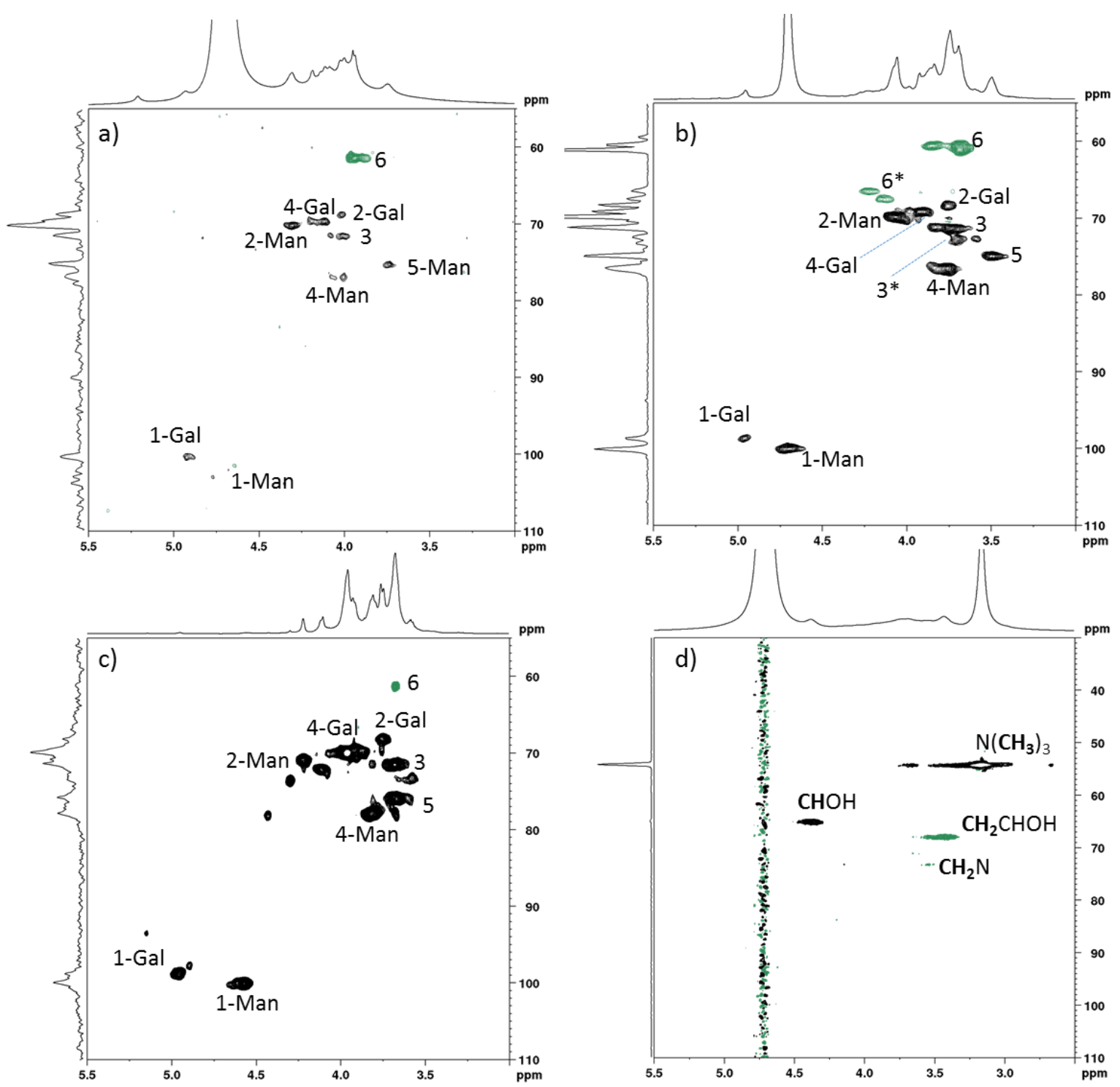

440 Figure $3-{ }^{1} \mathrm{H} /{ }^{13} \mathrm{C}$ HSQC-DEPT spectra of (a) LBG, (b)LBGS-M2-B1, (c) LBGC, and (d) LBGA; (*) Assignments attributed to the sulfate derivative.

The attachment of sulfate groups to the hydroxyls results usually in downfield shifts of the carbons bearing the sulfates and the protons linked to them (Duus, Gotfredsen \&

445 Bock, 2000). LBG primary hydroxyl groups in C-6 position are clearly the most reactive towards the sulfation reaction, as would be expected. In LBGS (Figure 3-b), the C-6 resonances exhibit a downfield shift to $66.4 \mathrm{ppm}$, indicative of C-6 sulfation. It is also noticeable a sulfate introduction in position C-3. The lower steric hindrance in the branched galactose residues in comparison to the main chain mannose leads to the assumption that sulfate introduction would have taken place preferentially in the 
hydroxilated positions of the former, and as such 3-Gal would have been preferentially substituted (Muschin et al., 2016). The carboxylation of LBG imposes a different effect on the ${ }^{1} \mathrm{H} /{ }^{13} \mathrm{C}$ HSQC-DEPT spectra, since the resonances of the positions that indeed react are expected to disapeer from the original location. From Figure 3-c it is possible 455 to observe that $\mathrm{C}-6$ resonance is almost absent, which means that once again this was the reaction location. Unreacted positions C-2,C-3 and C-4 appear unchanged, while additional resonances appear downshifted from the original envelope of resonances, most likely due to oxidized C-6 positions. The carboxylation is also confirmed by the presence of the carbonyl in the ${ }^{13} \mathrm{C}$ NMR spectra at $175.3 \mathrm{ppm}$ (Figure S2-f). The 460 attachment of $N$-(2-hydroxypropyl)- $N, N, N$-trimethylammonium to LBG produces a derivative with a high swelling capability. Here, the introduction of the ammonium group is confirmed by the well resolved $\mathrm{N}$-methyl resonances at $3.146 \mathrm{ppm}$. However, these resonances dominate the whole spectrum (Figure 3-d) and all ${ }^{1} \mathrm{H}$ signals are very broadned. As such, this sample was analysed in the solid state (Figure S2-e), where the 465 resonances of the ammonium group at $54.62 \mathrm{ppm}$, the anomeric carbons (102.17 and $100.71 \mathrm{ppm}$ ) and the remaining polysaccharide chain between 90 and $60 \mathrm{ppm}$ could be detected.

The average molecular weights, polydispersity index $(\mathrm{PdI})$, and radius of gyration $(R \mathrm{~g})$ of LBG and its derivatives are presented in Table S2. For the parent polysaccharide 470 (LBG), these are in general agreement with the literature (Dakia, Blecker, Robert, Whatelet \& Paquot, 2008; Kawamura, 2008). Upon chemical modification, an increase in both molecular weight and $R_{\mathrm{g}}$ was observed in LBGA, and a big decrease in these parameters was patent in LBGC and in the analysed sample of LBGS-M1. The increase identified in LBGA is attributable to the presence of the introduced pendant chains,

475 which led to an increase in the molar mass of the repeating unit and force the polymer, 
once in solution, and similarly to what happens in the crystalline state (XRD results), to adopt a conformation that is suitable to accommodate such bulky groups. The results observed in the LBGC and LBGS-M1 derivatives suggest the occurrence of depolymerization during the chemical modification, a common observation when the 480 conditions of either the oxidation (Cunha, Maciel, Sierakowski, Paula \& Feitosa, 2007) or the sulfation reaction (Alban, Schauerte \& Franz, 2002) are applied. The latter was already stated in a similar modification performed in pullulan (Dionísio, Braz, Corvo, Lourenço, Grenha \& da Costa). Moreover, at least in the analysed sample, and as verified in the referred sulfation of pullulan, no additional dehydration reactions, with 485 intra- and/or intermolecular crosslinking leading to a fraction of high molecular weight chains, observed in sulfation reactions carried out at higher temperatures (Mihai, Mocanu \& Carpov, 2001), occurred in this case.

Figure 4 shows the XRD patterns of the pristine and modified LBG samples. The pattern of LBG, with a broad peak centered at $c a .20^{\circ} 2 \theta$ with shoulders at $c a .7 .5$ and $15^{\circ} 2 \theta$, reflects the predominantly amorphous nature of the material. These shoulders vanish in the pattern of LBG modified with sulfate (LBGS-M2-B2), probably due to some changes in the organization of the polymer chains imposed by the sulfate groups. In what concerns the ammonium derivative, the pattern clearly shows an increase of intensity for higher d-spacings, which is compatible with an increase of the distance between the polymer chains, due to the long chain bearing the ammonium group (Dionísio, Braz, Corvo, Lourenço, Grenha \& da Costa). When compared with the other modifications, the introduction of carboxylate groups gives rise to the highest degree of disruption of the long-range order of the LBG polymer chains. The intensity of the peak that appears at $20^{\circ} 2 \theta$ in the pattern of the original polymer (LBG) is substantially

500 reduced and new broad peaks are now present at ca. 12 and $25^{\circ} 2 \theta$. This is not 
surprising, as the conversion of galactose and mannose units into the corresponding uronic acids would enormously affect the conformation of the polysaccharide chains and, consequently, the way they pack in the solid phase.

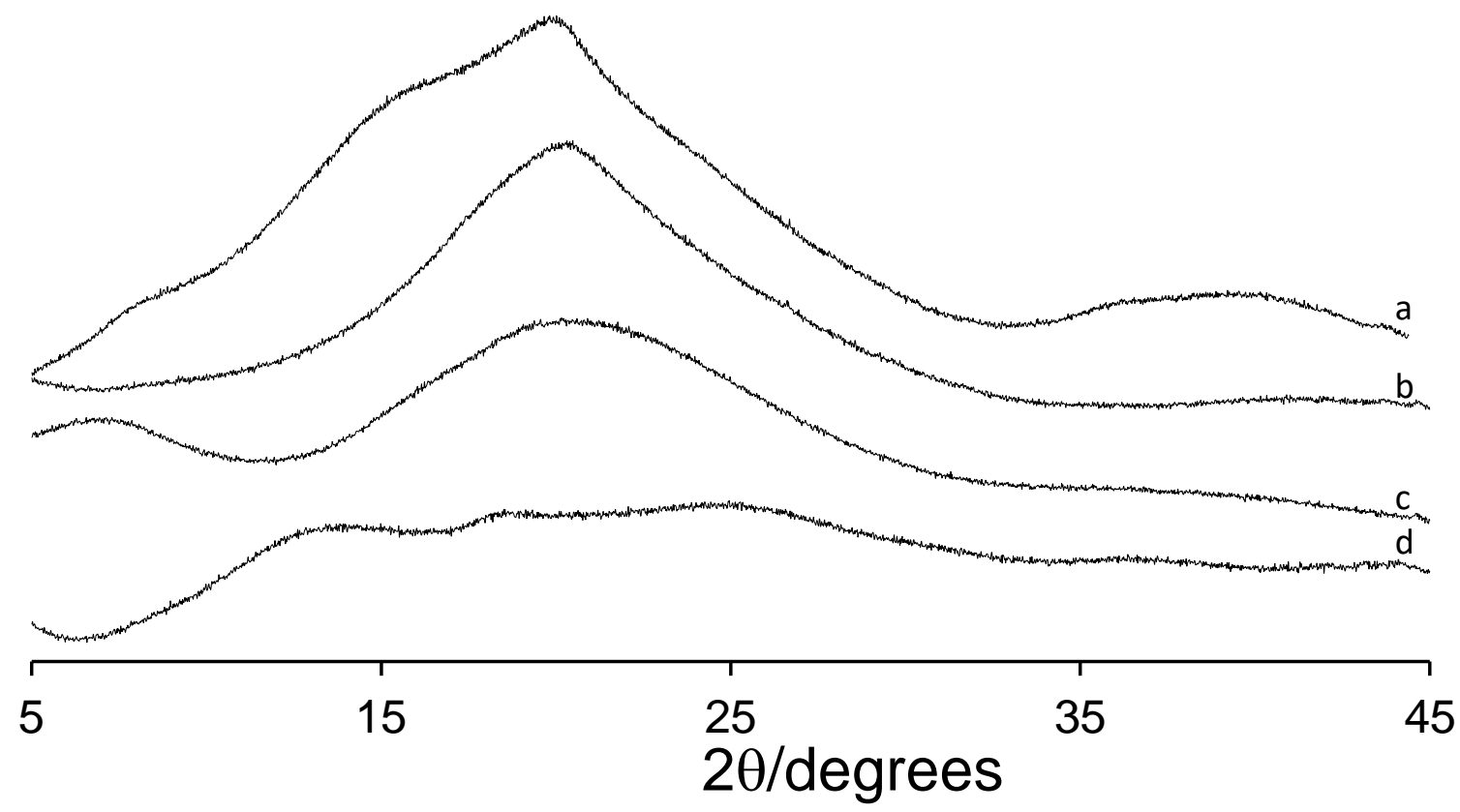

Figure 4 - XRD patterns of (a) pristine locust bean gum (LBG), (b) LBGS-M2-B2, (c) LBGA, and (d) LBGC.

\subsection{Characterization of nanoparticles}

510 The production of LBG derivatives described above endowed the polymer with charged groups, enabling the preparation of nanoparticles by polyelectrolyte complexation. This is a mild method occurring in hydrophilic medium, devoid of aggressive conditions such as organic solvents or high shear forces, and involving electrostatic interactions between oppositely charged polymers (Grenha, 2012; Prego, Torres \& Alonso, 2005).

515 Three derivatives were synthesized which were used in the production of different formulations of nanoparticles. The negatively charged sulfate and carboxylate derivatives were complexed with chitosan to produce CS/LBGS and CS/LBGC 
nanoparticles, respectively. In turn, the ammonium derivative was complexed with the sulfate derivative in the innovative approach of producing LBG-only nanoparticles referred nanoparticle formulations are displayed and discussed below.

\subsubsection{CS/LBGS and CS/LBGC nanoparticles}

The first approach towards the formulation of CS/LBGS and CS/LBGC nanoparticles involved the production of carriers having higher or at least the same amount of LBG derivative comparing to chitosan. In this regard, the starting mass ratios selected for the production of the referred formulations of nanoparticles were $1: 1,1: 1.5$ and $1: 2$. In the course of the experiments, the need to test other ratios was identified, not necessarily being coincident for each formulation, thus justifying the slight differences observed between the two formulations.

Table 1 displays the physicochemical characteristics of CS/LBGS nanoparticles. For the production of these nanoparticles, LBGS corresponding to method 1 was used. With CS/LBGS mass ratios varying between 1:1 and 1:2.5, and recalling that CS amount remains constant in all formulations, it was verified that nanoparticle size generally increased with increasing amounts of LBGS. The minimum size was $364 \mathrm{~nm}$ (CS/LBGS $=1: 1, \mathrm{w} / \mathrm{w})$ and the highest size was $589 \mathrm{~nm}(\mathrm{CS} / \mathrm{LBGS}=1: 2.5, \mathrm{w} / \mathrm{w})(P<0.05)$. 
Table 1 - Physicochemical characteristics and production yield of CS/LBGS, CS/LBGC and LBGA/LBGS unloaded nanoparticles (mean $\pm \mathrm{SD} ; \mathrm{n} \geq 3$ ). Different letters represent significant differences in each parameter and formulation $(P<0.05)$.

\begin{tabular}{|c|c|c|c|c|c|}
\hline Formulation & $\begin{array}{l}\text { Ratio } \\
(w / w)\end{array}$ & $\begin{array}{l}\text { Size } \\
(\mathbf{n m})\end{array}$ & PdI & $\begin{array}{c}\text { Zeta potential } \\
\qquad(\mathbf{m V})\end{array}$ & $\begin{array}{l}\text { Production } \\
\text { yield (\%) }\end{array}$ \\
\hline \multirow{5}{*}{ CS/LBGS } & $1: 1$ & $364.1 \pm 30.0^{\mathrm{a}}$ & $0.34 \pm 0.09$ & $+45.6 \pm 1.2^{\mathrm{d}}$ & $37.3 \pm 5.6^{\mathrm{h}}$ \\
\hline & $1: 1.25$ & $403.7 \pm 37.7^{a b}$ & $0.40 \pm 0.06$ & $+40.0 \pm 0.8^{\mathrm{e}}$ & $58.1 \pm 2.7^{\mathrm{i}}$ \\
\hline & $1: 1.5$ & $\mathrm{pp}^{*}$ & $1.0 \pm 0.0$ & $-5.9 \pm 4.4^{f}$ & n.d. \\
\hline & $1: 2$ & $500.3 \pm 59.6^{\mathrm{bc}}$ & $0.47 \pm 0.08$ & $-23.9 \pm 2.7^{g}$ & $56.6 \pm 7.2^{\mathrm{i}}$ \\
\hline & $1: 2.5$ & $589.0 \pm 69.5^{\mathrm{c}}$ & $0.54 \pm 0.03$ & $-28.5 \pm 5.0^{g}$ & n.d. \\
\hline \multirow{6}{*}{ CS/LBGC } & 1:0.75 & $489.9 \pm 63.6^{a}$ & $0.45 \pm 0.04$ & $+45.5 \pm 13.0^{b}$ & $49.0 \pm 5.0^{d}$ \\
\hline & 1:1 & $479.1 \pm 30.8^{a}$ & $0.51 \pm 0.07$ & $+42.2 \pm 7.4^{b}$ & $54.3 \pm 7.0^{\mathrm{d}}$ \\
\hline & $1: 1.25$ & $828.8 \pm 299.8^{a}$ & $0.64 \pm 0.15$ & $+28.8 \pm 7.3^{b}$ & n.d. \\
\hline & $1: 1.5$ & $\mathrm{pp}^{*}$ & $1.0 \pm 0.0$ & $-2.5 \pm 8.3^{c}$ & n.d. \\
\hline & $1: 2$ & $\mathrm{pp}^{*}$ & $1.0 \pm 0.0$ & $-15.2 \pm 7.4^{c}$ & n.d. \\
\hline & $1: 2$ & $206.6 \pm 5.0^{\mathrm{a}}$ & $0.13 \pm 0.03$ & $-27.8 \pm 1.4^{\mathrm{c}}$ & $30.0 \pm 8.6^{\mathrm{e}}$ \\
\hline \multirow[t]{2}{*}{ LBGA/LBGS } & 1:1 & $\mathrm{pp}$ & - & - & - \\
\hline & $2: 1$ & $368.3 \pm 19.3^{b}$ & $0.38 \pm 0.05$ & $+48.1 \pm 1.5^{\mathrm{d}}$ & $16.7 \pm 3.8^{f}$ \\
\hline
\end{tabular}

The registered increase in size as higher amount of LBGS is included in the formulations as compared with CS, might be explained by the increase of total mass of polymers that is present. This effect was also reported in other works using the same 550 nanoparticle production method (Grenha et al., 2010; Rodrigues, da Costa \& Grenha, 2012). Precipitation was found to occur for an intermediate formulation (CS/LBGS = $1: 1.5, \mathrm{w} / \mathrm{w})$, being coincident with a zeta potential close to zero $(-5.9 \mathrm{mV})$ that possibly 
is not sufficient to provide particle repulsion, thus leading to aggregation. A clear Tyndall effect was observed in all the other nanoparticle formulations. The formulations 1:1 and 1:1.25 (w/w) exhibited a strong positive zeta potential of more than $+40 \mathrm{mV}$. The incorporation of a higher amount of LBGS, from formulation 1:1 to 1:1.25 (w/w) resulted in a corresponding decrease in the zeta potential from $+46 \mathrm{mV}$ to $+40 \mathrm{mV}(P<$ 0.05). The formulations $1: 2$ and 1:2.5 (w/w) presented a complete shift in the zeta potential as the nanoparticles became negatively charged, with zeta potential reaching $29 \mathrm{mV}$. Again, the incorporation of a higher amount of LBGS led to a nominal decrease in the zeta potential, although this is not statistically significant. This absolute shift of nanoparticle charge reflects the higher amount of LBGS that is present in the nanoparticles, but also demonstrates that both polymers have different charge density. Zeta potential results are perfectly in line with the charge ratios that were calculated for each formulation of nanoparticles, as is depicted in Figure 5-a. This figure shows the effect of charge ratios on the zeta potential of CS/LBGS nanoparticles prepared with varying polymeric ratios. 


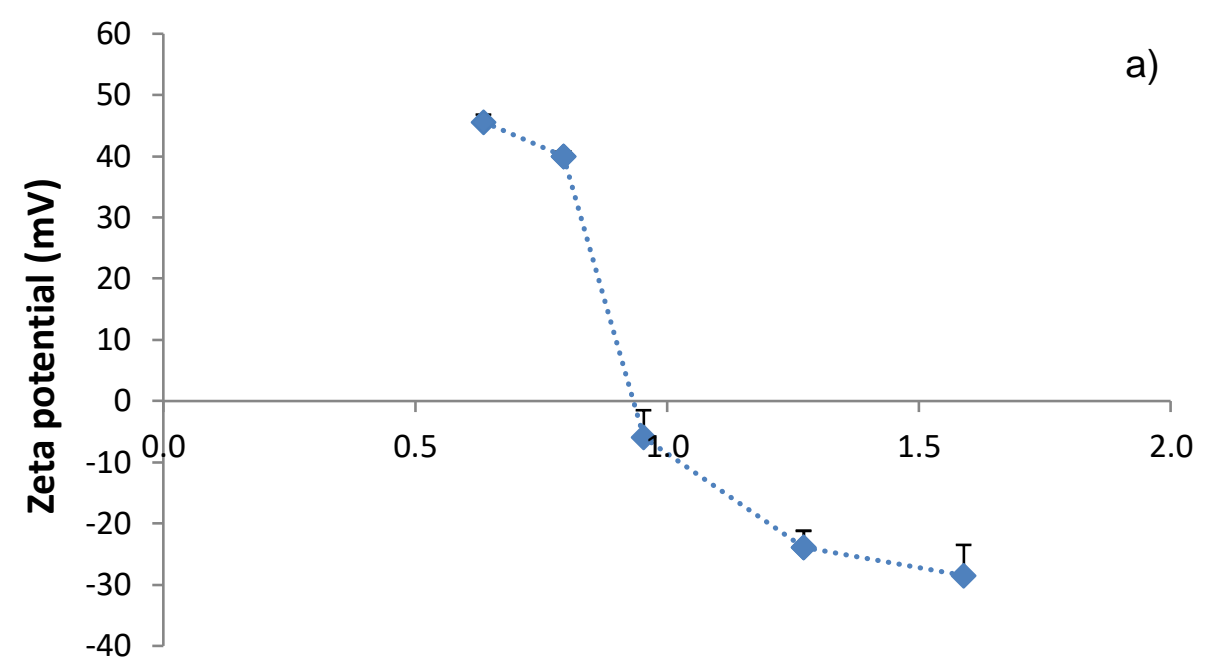

Charge ratio (-/+)

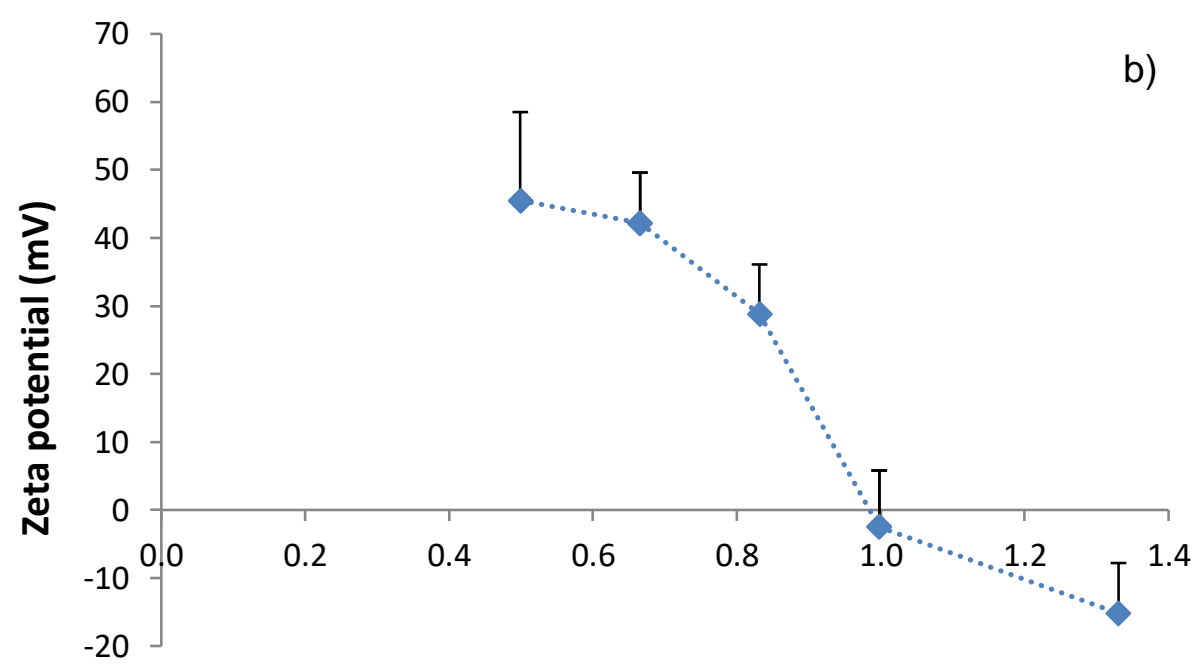

Charge ratio $(-/+)$

Figure 5 - Effect of charge ratio (-/+) on the zeta potential of (a) CS/LBGS nanoparticles and (b) CS/LBGC nanoparticles.

575 For each polymer, by dividing the charge of the repeating unit by its molar mass, a charge per mass ratio may be obtained. CS has higher charge per mass ratio than LBGS (4.72 $\times 10^{-3}$ vs $3.00 \times 10^{-3}$ charges/g, respectively), which justifies why formulations $\mathrm{CS} / \mathrm{LBGS}=1: 1$ and 1:1.25 $(\mathrm{w} / \mathrm{w})$ have a $-/+$ charge ratio below 1 . The strong positive zeta potential $(>+40 \mathrm{mV})$ of these nanoparticles is due to the predominance of positive charges. In turn, the occurrence of precipitation in the formulation 1:1.5 (w/w) was 
coincident with a charge ratio around 1 , justifying that the determined zeta potential was close to neutrality. In fact, although a 1:1 -/+ charge stoichiometry might not imply the occurrence of complete charge neutralization, due to steric limitations and different charge spacing in the intervenient species (Rodrigues, da Costa \& Grenha, 2012), one may assume a preferential interaction between the sulfate and the ammonium groups, both weakly hydrated, instead of with the strongly hydrated counterions (Crouzier \& Picart, 2009). This mainly leads to an intrinsic charge match in detriment of an extrinsic charge compensation and, thus, to a small deviation from neutrality. Finally, the continued addition of the negative polymer (formulations CS/LBGS $=1: 2$ and 1:2.5, $590 \mathrm{w} / \mathrm{w})$ produced an excess of negative charges, resulting in $-/+$ charge ratio above 1 and, consequently, negatively charged nanoparticles. A similar behavior concerning the charge ratios leading to either precipitation or formation of nanoparticles, was previously described (Rodrigues, da Costa \& Grenha, 2012).

The polydispersity index varied between 0.3 and 0.5 , which is considered high.

595 Regarding the production yield, very reasonable values for this nanoparticle production methodology, were obtained. A yield of $37 \%$ was registered for formulation 1:1 (w/w) which increased to $58 \%(P<0.05)$ for formulation $1: 1.25(\mathrm{w} / \mathrm{w})$. This is a result of the proper mechanism of nanoparticle formation, based on the neutralization of chitosan amino groups by the sulfate groups of LBGS. The incorporation of a higher amount of

600 LBGS provides an additional amount of sulfate groups that interacted with chitosan, thus forming a higher amount of nanoparticles (Fernández-Urrusuno, Romani, Calvo, Vila-Jato \& Alonso, 1999). However, this effect occurs up to a certain limit. As observed, further increasing the amount of LBGS led to precipitation, certainly because of the demonstrated neutralization of charges, as referred above. On keeping increasing 
605 LBGS mass, nanoparticles are again formed (CS/LBGS 1:2 and 1:2.5, w/w), this time with an opposite charge and a high yield (57\% for formulation $1: 2, \mathrm{w} / \mathrm{w})$.

The results obtained for $\mathrm{CS} / \mathrm{LBGC}$ nanoparticles were rather different comparing to those described above regarding CS/LBGS formulations. In this case, as shown in Table 1, the initially approached formulation of CS/LBGC 1:1(w/w) resulted in a size 610 of $479 \mathrm{~nm}$, which is more than $30 \%$ higher than the corresponding CS/LBGS formulation $(P<0.05)$. The formulation 1:1.5 (w/w) already presented precipitation, similarly to $1: 2(\mathrm{w} / \mathrm{w})$ and, therefore, the intermediate formulation $1: 1.25(\mathrm{w} / \mathrm{w})$ was produced.

The registered size revealed a strong increase to $829 \mathrm{~nm}$, although this is not statistically 615 significant as is accompanied by an extremely high standard deviation, which indicates reproducibility issues. This formulation also presented a high polydispersity index and, thus, was not characterized for production yield. An attempt was also performed to produce nanoparticles at CS/LBGC ratio of 1:0.75 (w/w), but the characteristics were very similar, under all aspects, to those of ratio $1: 1(\mathrm{w} / \mathrm{w})$. The polydispersity index was 620 around $0.5-0.6$, which is even higher than those registered for CS/LBGS nanoparticles, reinforcing the difficulty in producing suitable nanoparticles with the LBGC derivative. The zeta potentials were highly positive (around $+45 \mathrm{mV}$ ), which probably contributes to the system stability. The determination of the charge ratios involved in each formulation of nanoparticles is depicted in Figure 5-b.

625 As observed, formulations 1:0.75 and 1:1 (w/w) have a -/+ charge ratio between 0.5 and 0.7 which does not translate into significant differences in the zeta potential. Nanoparticles 1:1.25 (w/w) displayed a $-/+$ charge ratio of 0.85 which induced a nominal decrease of the zeta potential to $+29 \mathrm{mV}$, although not to a statistically significant level. As observed above for CS/LBGS nanoparticles, reaching a -/+ charge 
630 ratio around 1 (formulation 1:1.5, w/w) resulted in precipitation. However, in this case the continued addition of the negative polymer to formulate CS/LBGC $=1: 2(\mathrm{w} / \mathrm{w})$ nanoparticles still resulted in precipitation, despite the $-/+$ charge ratio of 1.4 . It is important to highlight that, while the resulting zeta potential for this formulation was of $-15 \mathrm{mV}$, in the CS/LBGS corresponding formulation was $-24 \mathrm{mV}$, which possibly

635 permitted enough repulsion to stabilize the formed nanoparticles. The determined production yields were satisfactory for this methodology, as referred above, being around 50\%. When comparing the zeta potentials of these nanoparticles with those obtained for CS/LBGS nanoparticles (Table 1), a similar trend was observed. In this regard, increasing the amount of LBGC present in the formulation

640 reflected in a decrease of the surface charge, owing to the higher amount of negative groups being incorporated. Similarly to CS/LBGS nanoparticles, the formulation 1:1.5 was the one showing neutrality (zeta potential of $-2.5 \mathrm{mV}$ ) and the further incorporation of LBGC led to a decrease in the surface charge. The precipitation verified for the latter was possibly due to the fact that the existing surface charges were not sufficient to

645 ensure particle repulsion. The resemblance of the trend, particularly regarding the shift of the zeta potential (occurring for mass ratio of 1:1.5), suggests the similarity of charge density in both derivatives. In fact, LBGS has a charge per mass ratio of $3.00 \times 10^{-3}$ charges/g, as stated before, and LBGC has $3.14 \times 10^{-3}$ charges $/ g$.

\subsubsection{LBGA/LBGS nanoparticles}

One of the great novelties of producing LBG charged derivatives was the possibility of using these to produce, for the first time, LBG-only nanoparticles. Given the difficulties in producing nanoparticles with the LBGC derivative, as stated above, it was decided to produce the LBG-only nanoparticles using just LBGS as negative counterpart. The 
655 nanoparticles were produced by complexation of this derivative (method $2-50 / 50$ mixtures of batches 2 and 3) with the ammonium derivative (LBGA) by the same methodology reported in the other cases (polyelectrolyte complexation).

After observing the precipitation of the formulation LBGA/LBGS 1:1 (w/w), possibly resulting from a (-/+) charge ratio of 1.09, formulations 1:2 (w/w) and 2:1 (w/w) were

660 developed, which results are depicted in Table 1.

The formulation containing the highest amount of LBGS registered size of $207 \mathrm{~nm}$ and low polydispersity index of 0.13 . Naturally, the zeta potential was negative $(-28 \mathrm{mV})$, reflecting the higher content of negatively charged derivative, which translated into a (/+) charge ratio of 2.17. As expected, the formulation having more LBGA exhibited a

665 strongly positive zeta potential $(+48 \mathrm{mV} ; P<0.05)$, as a result of the $(-/+)$ charge ratio of 0.54 . However, this particular formulation presented higher size $(368 \mathrm{~nm})$ along with higher polydispersity index $(P<0.05)$. At a first evaluation, the size differences could be considered unexpected. In fact, for the preparation of these nanoparticles, LBGA is kept constant at $0.5 \mathrm{mg} / \mathrm{mL}$ and LBGS concentration is adapted to meet the desired 670 ratio. Therefore, formulation 1:2 (w/w) accounts with a total polymeric mass of $1.5 \mathrm{mg}$, while formulation 2:1 (w/w) accounts with $0.75 \mathrm{mg}$. In line with this, formulation 1:2 (w/w) was perhaps expected to have larger size. However, if one considers the molecular weight of the derivatives, reported in section 3.3.1, LBGA has much higher $M_{\mathrm{n}}$ than LBGS (500 600 vs 21 380). In this regard, it becomes justifiable that 675 nanoparticles having double amount of LBGA comparing with LBGS are those displaying the highest size.

Regarding the production yield, this was very different between the two formulations. While formulation 1:2(w/w) resulted in $30 \%$, formulation $2: 1(\mathrm{w} / \mathrm{w})$ presented $17 \%(P$ $<0.05)$. This difference is probably due to variances in the molecular weight of the two 
derivatives. In formulation 1:2(w/w), there is a determined amount of a high molecular weight polymeric chain and a double amount of a shorter macromolecule that possibly presents higher diffusion. On the contrary, in formulation 2:1 (w/w) the amount of the polymer with higher molecular weight is double comparing with that of the smaller polymer, thus resulting in a lower number of interactions and limiting the amount of nanoparticles formed.

LBG-only nanoparticles were morphologically characterized by TEM and the specific formulation LBGA/LBGS 1:2 (w/w) was considered representative for this end. As shown in Figure 6, nanoparticles present a spherical shape and have compact structure.

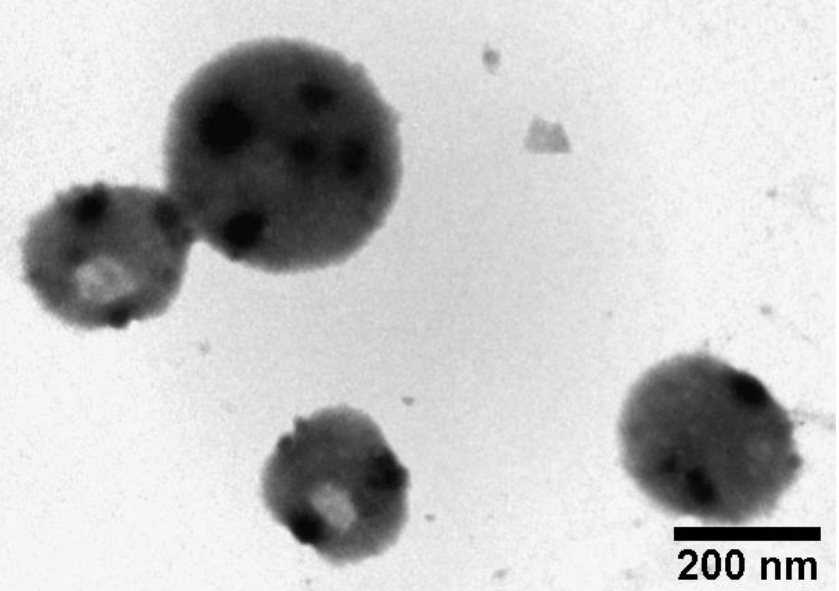

690 Figure 6 - TEM microphotograph of LBGA/LBGS = 1:2 (w/w) nanoparticles.

\subsection{Safety evaluation}

Caco-2 cells were used to evaluate the toxicological profile of LBG and the synthesized derivatives. Cell viability was determined after exposure to the mentioned materials at 695 different concentrations, for a period of $3 \mathrm{~h}$ (Figure S3) and $24 \mathrm{~h}$ (Figure 7). Cell viability values were calculated in relation to the $100 \%$ cell viability considered for the incubation with DMEM (negative control of cell death). The evaluation of LBG-based samples generally evidenced a mild effect on cell viability, considered to be devoid of 
biological relevance. In fact, with the exception of LBGA, all the other samples resulted in viabilities above $70 \%$ after $3 \mathrm{~h}$ or $24 \mathrm{~h}$ of exposure, when tested at concentrations varying within 0.1 and $1.0 \mathrm{mg} / \mathrm{mL}$. While at $3 \mathrm{~h}$ values remained above $88 \%$ in all conditions, the prolonged exposure until $24 \mathrm{~h}$ induced slight alterations. However, these were in most cases devoid of physiological relevance and the only remarkable effect resides in the decrease of the viability induced by the contact with LBGC at the highest concentration tested $(1.0 \mathrm{mg} / \mathrm{mL})(P<0.05)$ to a value around $70 \%$. Importantly, this is the value considered by ISO 10993-5 (ISO, 2009) as the level below which a toxic effect is assumed to occur.

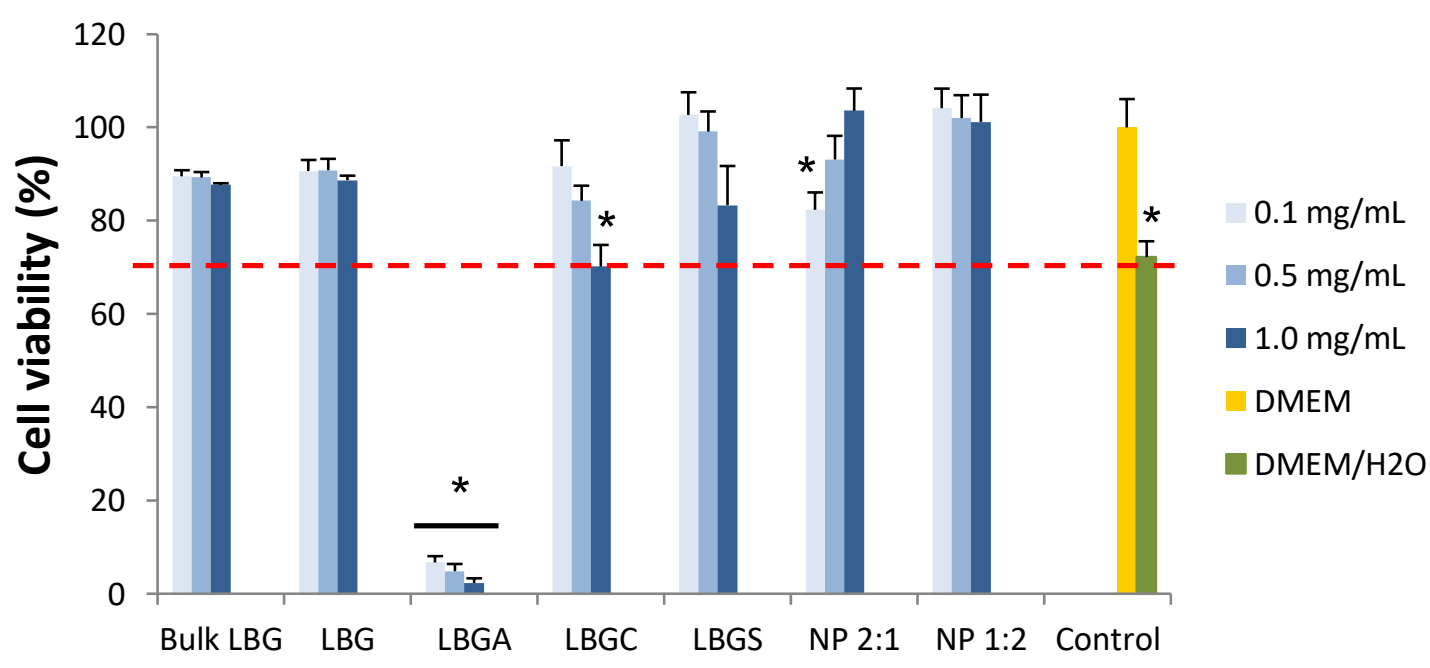

710 Figure 7 - Caco- 2 cell viability measured by the MTT assay after $24 \mathrm{~h}$ exposure to increasing concentrations of bulk Locust Bean Gum, purified Locust Bean Gum (LBG) and its ammonium (LBGA), carboxylate (LBGC) and sulfate (LBGS) derivatives; and LBGA/LBGS nanoparticles (NP). Data represent mean \pm SEM $(n \geq 3$, six replicates per experiment at each concentration). Dashed line indicates $70 \%$. $* P<0.05$ compared 715 with DMEM.

Although not directly proposed herein as matrix material per se, unmodified LBG was also tested, because its application in drug delivery has been reported, in many occasions addressing oral delivery strategies (Colombo et al., 1990; Conte \& Maggi, 1996; Coviello, Alhaique, Dorigo, Matricardi \& Grassi, 2007; Dey, Sa \& Maiti, 2015; 
Jana, Gandhi, Sheet \& Sen, 2015; Malik, Arora \& Singh, 2011a; Malik, Arora \& Singh, 2011b; Ngwuluka, Choonara, Kumar, du Toit, Modi \& Pillay, 2015; Sandolo, Coviello, Matricardi \& Alhaique, 2007; Sujja-areevath, Munday, Cox \& Khan, 1998; Syed, Mangamoori \& Rao, 2010; Tobyn, Staniforth, Baichwal \& McCall, 1996), but data on 725 its effect on epithelial cells are not available in the literature. Moreover, a comparison between bulk LBG and purified LBG was performed, revealing no significant differences, which indicates an absence of effect of the purification process in the cytotoxic profile of the material. It is important to mention that the results shown for LBGS sample correspond to the derivative obtained by the second method of synthesis

730 (method 2 - batch 1), which were similar to those registered for the derivative obtained in the first method (method 1; data not shown).

As mentioned before, LBGA is the material that presents the most distinct behavior, appearing as the exception to the mild effect observed for the tested materials. In fact, a strong decrease of cell viability to approximately $30 \%$ was obtained for all the tested

735 concentrations even upon 3 h exposure (Figure S3). The effect was even more drastic after 24 h (Figure 7), when a very low level of cell survival was registered $(P<0.05)$. Regarding concentration, there are no evidences of statistically significant concentration-dependent effect. The influence of surface charge on cytotoxicity remains largely unresolved and sometimes the literature reports contradictory results. This is

740 possibly due to different characteristics of basic materials being used and also to dissimilar assay conditions, which are frequently not described in sufficient detail. Nevertheless, there are many indications suggesting that surface charge has a role on cellular uptake (Fröhlich, 2012; Zhao, Zhao, Liu, Chang, Chen \& Zhao, 2011) and on the toxicological effect of substances. In this context, positively charged materials have 745 been frequently found to be more cytotoxic than neutral or negatively charged 
counterparts, because positive charges provide a means for stronger interaction with cell surfaces, in many cases associated with internalization of the material (Bhattacharjee et al., 2010; Fröhlich, 2012; Ilinskaya, Dreyer, Mitkevich, Shaw, Pace \& Makarov, 2002; Platel, Carpentier, Becart, Mordacq, Betbeder \& Nesslany, 2016; Turcotte, Lavis \& 750 Raines, 2009). These statements are coincident with the results of our work, since the neutral (bulk LBG and LBG) and negatively charged materials (LBGC and LBGS) were devoid of toxicity. Another parameter that could be indicated as playing a significant role on toxicity consists on the molecular weight of the polymers. In this regard, although it could be suggested that smaller sizes have higher probability to be internalized by the cells, the literature has been reporting no correlation (Huang, Khor \& Lim, 2004). In this work, the molecular weight of the polymers also seems to not be driving the cytotoxic behaviour, as LBGS is the smallest molecule and shows no toxic effect.

Comparing to LBGA, a very similar toxicological profile was observed for an

760 ammonium derivative of another polysaccharide, pullulan, which was synthesized using the same methodology (Dionísio, Braz, Corvo, Lourenço, Grenha \& da Costa, 2016; Dionísio, Cordeiro, Remuñán-López, Seijo, Rosa da Costa \& Grenha, 2013). In that case, the assessment was performed in Calu-3 cells (bronchial cell line) and cell viabilities around 50-60\% were observed after $3 \mathrm{~h}$, decreasing to $40 \%$ at $24 \mathrm{~h}$. Although 765 a time-dependent effect is also clearly observed, the effect on cell viability is not as strong as for LBGA. The first consideration to take into account is the fact that the assessment was performed in different cell lines, which may translate into different sensitivity. Additionally, different charge density of the polymers might be indicated as possible justification. In this regard, LBGA has a DS of 4.24, while the corresponding 770 pullulan derivative (ammonium pullulan) has a DS of 2 (Dionísio, Braz, Corvo, 
Lourenço, Grenha \& da Costa). A higher number of positive charges results in stronger interactions and, thus, in lower cell viability. Complementing this idea, a work reporting the cytotoxic effect of cationic pullulan microparticles on human leukemic K562(S) cells, has established that toxicity increased with the increase molar concentration of amino groups (Constantin, Fundueanu, Cortesi, Esposito \& Nastruzzi, 2003). In the work reporting the cytotoxic evaluation of pullulan derivatives, a sulfate derivative of that polysaccharide was also assessed. Similarly to what was observed for LBGS, the registered cell viability was well above 80\% (Dionísio, Cordeiro, Remuñán-López, Seijo, Rosa da Costa \& Grenha, 2013).

780 Considering that polymer samples were solubilized in water and diluted with cell culture medium prior to incubation with the cells, an additional control was performed consisting in a mixture of DMEM and $\mathrm{H}_{2} \mathrm{O}$ in the same ratio used for the samples. This enables a real evaluation of the contribution of the polymers to the final cell viability. The cell viability induced by this control varied between $72 \%$ and $80 \%$. Upon $3 \mathrm{~h}$ of 785 contact there is a statistically significant difference between the control (DMEM $\left.+\mathrm{H}_{2} \mathrm{O}\right)$ and all samples, but with LBGS (Figure S3). In fact, higher cell viability is observed upon exposure to bulk LBG, LBG and LBGC, suggesting a positive effect of the presence of the polymers. Interestingly, after $24 \mathrm{~h}$ exposure, a shift is observed in the effect induced by LBGC and LBGS (Figure 7). In the former, the prolonged contact 790 with the cells at the two highest concentrations reverts the positive effect on cell viability observed at $3 \mathrm{~h}$. For LBGS, the results demonstrate that at the two lowest concentrations, the more prolonged contact improves cell viability, which was not registered at $3 \mathrm{~h}$.

One of the most important information provided by the evaluation performed with the 795 MTT assay, is that only the more prolonged exposure to the highest concentration tested 
$(1.0 \mathrm{mg} / \mathrm{mL} ; 24 \mathrm{~h})$ induced a relevant decrease of Caco-2 cell viability (exception for LBGA). Therefore, it was deemed important to complement the results at these conditions by means of the quantification of the amount of LDH released by Caco-2 cells. To perform this assay, DMEM was used as negative control of LDH release and a

800 lysis buffer was used as positive control. Thus, the negative control (DMEM) corresponds to a normal cell death, while the positive control (lysis buffer) represents $100 \%$ cell death.

The results of $\mathrm{LDH}$ release after $24 \mathrm{~h}$ exposure to the materials at the concentration of $1.0 \mathrm{mg} / \mathrm{mL}$ (Figure $\mathbf{S 4}$ ) showed no statistically significant differences between the 805 negative control (DMEM), bulk LBG, LBG, LBGC and LBGS. This means that these materials do not compromise Caco-2 cell membrane integrity, as LDH release was not increased when compared with that observed upon incubation with cell culture medium (DMEM). On the contrary, the contact with LBGA resulted in 90\% LDH release, which is considered comparable to that induced by the lysis buffer, thus indicating a high

810 cytotoxic effect that results in cell membrane disruption. The results obtained in this assay reinforce those found in the MTT tests, confirming the high cytotoxicity of LBGA.

Overall, the results obtained with these complementary cytotoxicity assays indicate that, with the exception of LBGA, LBG and negatively charged derivatives, present no

815 cytotoxicity towards this in vitro intestinal model. This was observed even for the highest concentration tested $(1.0 \mathrm{mg} / \mathrm{mL})$ and for prolonged contact $(24 \mathrm{~h})$, suggesting their relative safety for an application as matrix materials of oral drug delivery systems. Complementarily, the effect on cell viability provided by LBGS (method 2 - batch 1 ) was assessed in Calu-3 and A549 cells (respiratory epithelial cells) and the results are in 820 line with those observed for Caco-2 cells (Figures S5 and S6). 
Proposing materials for drug delivery applications requires testing the developed carriers and not only assume the apparent absence of cytotoxicity of the polymers. In this regard, it is consensual that carriers exhibit new and unique properties, thus generating potential different risks as compared to the raw materials of the same 825 chemistry (Aillon, Xie, El-Gendy, Berkland \& Forrest, 2009), as observed in other works (Dionísio, Braz, Corvo, Lourenço, Grenha \& da Costa, 2016; Dionísio, Cordeiro, Remuñán-López, Seijo, Rosa da Costa \& Grenha, 2013). In this regard, in addition to the evaluation of the polymer and the synthesized derivatives, a preliminary evaluation of LBG-based nanoparticles was further performed using the MTT assay. Although

830 several formulations were proposed and developed herein, that corresponding to LBGonly nanoparticles was selected for this step due to the novelty of the polymer in nanoparticle production.

The viability of Caco-2 cells upon exposure to LBGA/LBGS nanoparticles is shown in Figure S7 (3 h) and Figure 7 (24 h). The two formulations LBGA/LBGS 2:1 and 1:2

$835(\mathrm{w} / \mathrm{w})$ were assessed. For formulation 2:1 (w/w) the comparison of results obtained for each tested time revealed a statistically significant difference between concentrations 0.1 and $1.0 \mathrm{mg} / \mathrm{mL}(P<0.05)$. Formulation 1:2 (w/w) did not evidence significant differences between all concentrations at the two tested times. A similar observation was made after comparing the same concentrations for different times ( $3 \mathrm{~h}$ and $24 \mathrm{~h}$ ).

840 The most remarkable result is that no significant effect on cell viability is observed for both formulations at all concentrations, up to $24 \mathrm{~h}$. Actually, the registered viability was over $80 \%$ in all cases, which, as said before, is considered very acceptable according to the ISO10993-5 (ISO, 2009).

Curiously, the exposure of the cells to the formulation LBGA/LBGS 2:1 (w/w) resulted 845 in an increase of cell viability with the increase of nanoparticle concentration at $3 \mathrm{~h}$ and 
$24 \mathrm{~h}(P<0.05)$. This was unexpected and may be due to the fact that LBG is a polysaccharide with capacity to promote cell proliferation in some cell lines, as reported in the literature (Perestrelo, Grenha, Rosa da Costa \& Belo, 2014). Despite the formulation LBGA/LBGS 2:1 (w/w) could improve cell proliferation with increasing 850 concentrations, formulation LBGA/LBGS 1:2(w/w), generally induced constant cell viability near $100 \%$, irrespective of the concentration.

Comparing with the control (DMEM $\left.+\mathrm{H}_{2} \mathrm{O}\right)$ it is observed that the nanoparticles generally elicit higher cell viability, varying between $82 \%$ and $100 \%(P<0.05)$. The most remarkable observation in the whole set of cell viability assessment is that, in spite

855 of the strong decrease in cell viability induced by the contact with LBGA, this effect was completely reverted when the cells were exposed to a nanoparticulate form of the derivative. This was also observed in works using an ammonium derivative of pullulan, in which the derivative elicited around $40 \%$ cell viability upon $24 \mathrm{~h}$ of exposure, while nanoparticles produced with the polymer registered increased cell viabilities to values of $86070 \%$ - 80\% (Dionísio, Braz, Corvo, Lourenço, Grenha \& da Costa; Dionísio, Cordeiro, Remuñán-López, Seijo, Rosa da Costa \& Grenha, 2013). The different impact on cell viability generated by LBGA in form of polymer and of nanoparticles is possibly explained by a differential contact of each of the materials with the cells. While the polymer in form of a solute is presented as an extended chain and, thus, has a higher 865 surface of contact with the cells, nanoparticles have comparatively a lower contact. Additionally, the number of positive charges available for interaction with the negatively charged cells upon complexation with LBGS is significantly decreased, thus decreasing the potential toxicity (Huang, Khor \& Lim, 2004). This reinforces the need to evaluate separately the carriers and the raw materials, as the former may exhibit 
870 different properties, that may encompass different risks (Aillon, Xie, El-Gendy, Berkland \& Forrest, 2009).

As also observed for another formulation of LBG-based nanoparticles (CS/LBGS), which already shown to be promising for oral immunization (Braz, Grenha, Ferreira, Rosa da Costa, Gamazo \& Sarmento, 2017), these preliminary results suggest an

875 absence of overt toxicity of LBG-only nanoparticles, thus potentiating possible applications. Nevertheless, it is recognized that further studies need to be performed to reach a more accurate conclusion in this regard.

\section{Conclusions}

880 LBG demonstrated to be a good substrate for the production of charged derivatives, permitting the synthesis of ammonium, sulfated and carboxylated LBG. Several characterization techniques were used to confirm the presence of the new chemical groups introduced in each new derivative.

Using a method of polyelectrolyte complexation, the produced derivatives were applied

885 in the preparation of different formulations of LBG-based nanoparticles, reported herein for the first time. When the negatively charged derivatives (sulfated and carboxylated LBG) were used, chitosan was the applied positively charged polyelectrolyte. In turn, ammonium LBG was complexed with sulfated LBG to obtain LBG-only nanoparticles. The physicochemical characteristics of nanoparticles were highly dependent on their

890 composition and on the charge ratios applied in each complexation being performed. Generally, the observed characteristics, with sizes around 200-400 nm in certain cases, and tailorable zeta potential according to setup conditions, are suggested as adequate for drug delivery applications. 
A preliminary toxicological evaluation of LBG derivatives and the produced

nanoparticles was performed, assessing both the metabolic activity and the cell

membrane integrity of representative intestinal cells (Caco-2) after an exposure of up to

$24 \mathrm{~h}$ to concentrations as high as $1 \mathrm{mg} / \mathrm{mL}$. Severe cytotoxicity was found for the ammonium derivative of LBG, but this was clearly reverted after the assembly of nanoparticles, which evidenced a very mild effect on Caco-2 cell viability. The results

900 as a whole indicate the possibility to use the synthesized LBG derivatives to produce nanoparticles for drug delivery applications.

\section{Acknowledgements}

This work was supported by national Portuguese funding through FCT - Fundação para

905 a Ciência e a Tecnologia, project ref. PTDC/SAU-FCF/100291/2008, PEst-

OE/EQB/LA0023/2011, UID/QUI/00100/2013, UID/Multi/04326/2013, UID/BIM/04773/2013, UID/CTM/50025/2013 and PEst-OE/QUI/UI4023/2014.

This work was also financed by FEDER - Fundo Europeu de Desenvolvimento

Regional funds through the COMPETE 2020 - Operacional Programme for

910 Competitiveness and Internationalisation (POCI), Portugal 2020, and by Portuguese funds through FCT - Fundação para a Ciência e a Tecnologia/ Ministério da Ciência, Tecnologia e Inovação in the framework of the project "Institute for Research and Innovation in Health Sciences" (POCI-01-0145-FEDER-007274), POCI-01-0145FEDER-007688 and PTNMR 22161.

915

\section{References}

Aillon, K. L., Xie, Y., El-Gendy, N., Berkland, C. J., \& Forrest, M. L. (2009). Effects of nanomaterial physicochemical properties on in vivo toxicity. Advanced Drug Delivery Reviews, 61(6), 457466. 
920 Alban, S., Schauerte, A., \& Franz, G. (2002). Anticoagulant sulfated polysaccharides: Part I. Synthesis and structure-activity relationships of new pullulan sulfates. Carbohydrate Polymers, 47(3), 267-276.

Alonso, M. J. (2004). Nanomedicines for overcoming biological barriers. Biomedicine and Pharmacotherapy, 58, 168-172.

925 Antosova, Z., Mackova, M., Kral, V., \& Macek, T. (2009). Therapeutic application of peptides and proteins: parenteral forever? Trends in Biotechnology, 27(11), 628-635.

Arca, H. C., Gunbeyaz, M., \& Senel, S. (2009). Chitosan-based systems for the delivery of vaccine antigens. Expert Reviews of Vaccines, 8, 937-953.

Barak, S., \& Mudgil, D. (2014). Locust bean gum: Processing, properties and food applications -

930 A review. International Journal of Biological Macromolecules, 66, 74-80.

Bhattacharjee, S., de Haan, L., Evers, N., Jiang, X., Marcelis, A., Zuilhof, H., Rietjens, I., \& Alink, G. (2010). Role of surface charge and oxidative stress in cytotoxicity of organic monolayercoated silicon nanoparticles towards macrophage NR8383 cells. Particle and Fibre Toxicology, 7(25), 2-12.

935 Bhattarai, N., Gunn, J., \& Zhang, M. (2010). Chitosan-based hydrogels for controlled, localized drug delivery. Adv Drug Deliv Rev, 62(1), 83-99.

Bouzouita, N., Khaldi, A., Zgoulli, S., Chebil, L., Chekki, R., Chaabouni, M. M., \& Thonart, P. (2007). The analysis of crude and purified locust bean gum: A comparison of samples from different carob tree populations in Tunisia. Food Chemistry, 101(4), 1508-1515.

940 Braz, L., Grenha, A., Ferreira, D., Rosa da Costa, A. M., Gamazo, C., \& Sarmento, B. (2017). Chitosan/sulfated locust bean gum nanoparticles: In vitro and in vivo evaluation towards an application in oral immunization. International Journal of Biological Macromolecules, 96, 786797.

Casettari, L., \& Illum, L. (2014). Chitosan in nasal delivery systems for therapeutic drugs.

945 Journal of Controlled Release, 190, 189-200.

Colombo, P., Conte, U., Gazzaniga, A., Maggi, L., Sangalli, M. E., Peppas, N. A., \& La Manna, A. (1990). Drug release modulation by physical restrictions of matrix swelling. International Journal of Pharmaceutics, 63(1), 43-48.

Constantin, M., Fundueanu, G., Cortesi, R., Esposito, E., \& Nastruzzi, C. (2003). Aminated

950 polysaccharide microspheres as DNA delivery systems. Drug Delivery, 10(3), 139-149. Conte, U., \& Maggi, L. (1996). Modulation of the dissolution profiles from Geomatrix ${ }^{\circledR}$ multilayer matrix tablets containing drugs of different solubility. Biomaterials, 17(9), 889-896. Coviello, T., Alhaique, F., Dorigo, A., Matricardi, P., \& Grassi, M. (2007). Two galactomannans and scleroglucan as matrices for drug delivery: preparation and release studies. European

955 Journal of Pharmaceutics and Biopharmaceutics, 66(2), 200-209.

Crouzier, T., \& Picart, C. (2009). Ion pairing and hydration in polyelectrolyte multilayer films containing polysaccharides. Biomacromolecules, 10(2), 433-442.

Csaba, N., Garcia-Fuentes, M., \& Alonso, M. J. (2006). The performance of nanocarriers for transmucosal drug delivery. Expert Opinion on Drug Delivery, 3(4), 463-478.

960 Cunha, P. L. R., Maciel, J. S., Sierakowski, M. R., Paula, R. C. M. d., \& Feitosa, J. P. A. (2007). Oxidation of cashew tree gum exudate polysaccharide with TEMPO reagent. Journal of the Brazilian Chemical Society, 18, 85-92.

da Silva Perez, D., Montanari, S., \& Vignon, M. R. (2003). TEMPO-mediated oxidation of cellulose III. Biomacromolecules, 4(5), 1417-1425.

965 Dakia, P., Blecker, C., Robert, C., Whatelet, B., \& Paquot, M. (2008). Composition and physicochemical properties of locust bean gum extracted from whole seeds by acid or water dehulling pre-treatment. Food Hydrocolloids, 22, 807-818.

de la Fuente, M., Csaba, N., Garcia-Fuentes, M., \& Alonso, M. J. (2008). Nanoparticles as protein and gene carriers to mucosal surfaces. Nanomedicine, 3, 845-857. 
970 Dey, P., Sa, B., \& Maiti, S. (2015). Impact of gelation period on modified locust bean-alginate interpenetrating beads for oral glipizide delivery. International Journal of Biological Macromolecules, 76, 176-180.

Dionísio, M., Braz, L., Corvo, M., Lourenço, J. P., Grenha, A., \& da Costa, A. M. R. (2016). Charged pullulan derivatives for the development of nanocarriers by polyelectrolyte

975 complexation. International Journal of Biological Macromolecules, 86, 129-138. Dionísio, M., Cordeiro, C., Remuñán-López, C., Seijo, B., Rosa da Costa, A. M., \& Grenha, A. (2013). Pullulan-based nanoparticles as carriers for transmucosal protein delivery. European Journal of Pharmaceutical Sciences, 50(1), 102-113.

Dionísio, M., \& Grenha, A. (2012). Locust bean gum: exploring its potential for

980 biopharmaceutical applications. Journal of Pharmacy and Bioallied Sciences, 4(3), 75-85. Duus, J. Ø., Gotfredsen, C. H., \& Bock, K. (2000). Carbohydrate structural determination by NMR spectroscopy: modern methods and limitations. Chemical Reviews, 100(12), 4589-4614. Fernández-Urrusuno, R., Romani, D., Calvo, P., Vila-Jato, J., \& Alonso, M. (1999). Development of a freeze-dried formulation of insulin-loaded chitosan nanoparticles intended for nasal

985 administration. STP Pharma Sciences, 9, 429-436.

Fröhlich, E. (2012). The role of surface charge in cellular uptake and cytotoxicity of medical nanoparticles. International Journal of Nanomedicine, 7, 5577-5591.

Grenha, A. (2012). Chitosan nanoparticles: a survey of preparation methods. Journal of Drug Targeting, 20(4), 291-300.

990 Grenha, A., Gomes, M. E., Rodrigues, M., Santo, V. E., Mano, J. F., Neves, N. M., \& Reis, R. L. (2010). Development of new chitosan/carrageenan nanoparticles for drug delivery applications. Journal of Biomedical Materials Research Part A, 92A(4), 1265-1272.

Huang, M., Khor, E., \& Lim, L.-Y. (2004). Uptake and cytotoxicity of chitosan molecules and nanoparticles: Effects of molecular weight and degree of deacetylation. Pharmaceutical

995 Research, 21(2), 344-353.

Ilinskaya, O., Dreyer, F., Mitkevich, V., Shaw, K., Pace, C., \& Makarov, A. (2002). Changing the net charge from negative to positive makes ribonuclease Sa cytotoxic. Protein Science, 11(10), 2522-2525.

ISO. (2009). Biological evaluation of medical devices Part 5: Tests for in vitro cytotoxicity. (Vol.

1000 10993-5): International Organization for Standardization.

Jana, S., Gandhi, A., Sheet, S., \& Sen, K. K. (2015). Metal ion-induced alginate-locust bean gum IPN microspheres for sustained oral delivery of aceclofenac. International Journal of Biological Macromolecules, 72, 47-53.

Kadiyala, I., Loo, Y., Roy, K., Rice, J., \& Leong, K. W. (2010). Transport of chitosan-DNA

1005 nanoparticles in human intestinal M-cell model versus normal intestinal enterocytes. European Journal of Pharmaceutical Sciences, 39, 103-109.

Kammona, O., \& Kiparissides, C. (2012). Recent advances in nanocarrier-based mucosal delivery of biomolecules. Journal of Controlled Release, 161(3), 781-794.

Kawamura, Y. (2008). Carob Bean Gum Chemical and Technical Assessment. Joint FAO/WHO

1010 Expert Committee on Food Additives.

Lavelle, E. C., \& O'Hagan, D. T. (2006). Delivery systems and adjuvants for oral vaccines. Expert Opinion on Drug Delivery, 3, 747-762.

Mähner, C., Lechner, M. D., \& Nordmeier, E. (2001). Synthesis and characterisation of dextran and pullulan sulphate. Carbohydrate Research, 331(2), 203-208.

1015 Maiti, S., Chowdhury, M., Chakraborty, A., Ray, S., \& Sa, B. (2014). Sulfated locust bean gum hydrogel beads for immediate analgesic effect of tramadol hydrochloride. Journal of Scientific and Industrial Research, 73, 21-28.

Malik, K., Arora, G., \& Singh, I. (2011a). Locust bean gum as superdisintegrant - Formulation and evaluation of nimesulide orodispersible tablets. Polimery w Medycynie, 41(1), 17-28.

1020 Malik, K., Arora, G., \& Singh, I. (2011b). Taste masked microspheres of ofloxacin: Formulation and evaluation of orodispersible tablets. Scientia Pharmaceutica, 79(3), 653-672. 
Mihai, D., Mocanu, G., \& Carpov, A. (2001). Chemical reactions on polysaccharides: I. Pullulan sulfation. European Polymer Journal, 37(3), 541-546.

Mizrahy, S., \& Peer, D. (2012). Polysaccharides as building blocks for nanotherapeutics. The

1025 Chemistry Society Reviews, 41, 2623-2640.

Muschin, T., Budragchaa, D., Kanamoto, T., Nakashima, H., Ichiyama, K., Yamamoto, N., Shuqin, H., \& Yoshida, T. (2016). Chemically sulfated natural galactomannans with specific antiviral and anticoagulant activities. International Journal of Biological Macromolecules, 89, 415-420.

1030 Nakanishi, K., Goto, T., \& Ohashi, M. (1957). Infrared spectra of organic ammonium compounds. Bulletin of the Chemical Society of Japan, 30(4), 403-408.

Ngwuluka, N. C., Choonara, Y. E., Kumar, P., du Toit, L. C., Modi, G., \& Pillay, V. (2015). A coblended locust bean gum and polymethacrylate- $\mathrm{NaCMC}$ matrix to achieve zero-order release via hydro-erosive modulation. AAPS PharmSciTech, 16(6), 1377-1389.

1035 Perestrelo, A. R., Grenha, A., Rosa da Costa, A. M., \& Belo, J. A. (2014). Locust bean gum as an alternative polymeric coating for embryonic stem cell culture. Mater Sci Eng C Mater Biol Appl, 40, 336-344.

Platel, A., Carpentier, R., Becart, E., Mordacq, G., Betbeder, D., \& Nesslany, F. (2016). Influence of the surface charge of PLGA nanoparticles on their in vitro genotoxicity, cytotoxicity, ROS

1040 production and endocytosis. Journal of Applied Toxicology, 36(3), 434-444.

Pollard, M., Kelly, R., Wahl, C., Fischer, K. P., Windhab, E., Eder, B., \& Amadò, R. (2007).

Investigation of equilibrium solubility of a carob galactomannan. Food Hydrocolloids, 21, 683692.

Prajapati, V. D., Jani, G. K., Moradiya, N. G., Randeria, N. P., \& Nagar, B. J. (2013). Locust bean

1045 gum: A versatile biopolymer. Carbohydrate Polymers, 94(2), 814-821.

Prego, C., Torres, D., \& Alonso, M. J. (2005). The potential of chitosan for the oral administration of peptides. Expert Opinion on Drug Delivery, 2, 843-854.

Qin, C., Xiao, Q., Li, H., Fang, M., Liu, Y., Chen, X., \& Li, Q. (2004). Calorimetric studies of the action of chitosan-N-2-hydroxypropyl trimethyl ammonium chloride on the growth of

1050 microorganisms. International Journal of Biological Macromolecules, 34(1-2), 121-126. Rader, R. A. (2008). (Re)defining biopharmaceutical. Nature Biotechnology, 26(7), 743-751. Rekha, M. R., \& Sharma, C. P. (2009). Blood compatibility and in vitro transfection studies on cationically modified pullulan for liver cell targeted gene delivery. Biomaterials, 30(34), 66556664.

1055 Rodrigues, S., da Costa, A. M., \& Grenha, A. (2012). Chitosan/carrageenan nanoparticles: effect of cross-linking with tripolyphosphate and charge ratios. Carbohydrate Polymers, 89(1), 282289.

Sandolo, C., Coviello, T., Matricardi, P., \& Alhaique, F. (2007). Characterization of polysaccharide hydrogels for modified drug delivery. European Biophysics Journal, 36(7), 693-

1060700.

Sierakowski, M. R., Milas, M., Desbrières, J., \& Rinaudo, M. (2000). Specific modifications of galactomannans. Carbohydrate Polymers, 42, 51-57.

Simkovic, I., Yadav, M. P., Zalibera, M., \& Hicks, K. B. (2009). Chemical modification of corn fiber with ion-exchanging groups. Carbohydrate Polymers, 76, 250-254.

1065 Sudhakar, Y., Kuotsu, K., \& Bandyopadhyay, A. K. (2006). Buccal bioadhesive drug delivery - A promising option for orally less efficient drugs. Journal of Controlled Release, 114, 15-40.

Sujja-areevath, J., Munday, D. L., Cox, P. J., \& Khan, K. A. (1998). Relationship between swelling, erosion and drug release in hydrophillic natural gum mini-matrix formulations. European Journal of Pharmaceutical Sciences, 6, 207-217.

1070 Surana, S., Munday, D., Cox, P., \& Khan, K. (1998). Relationship between swelling, erosion and drug release in hydrophilic natural gum mini-matrix formulations. European Journal of Pharmaceutical Sciences, 6, 207-217. 
Syed, I., Mangamoori, L., \& Rao, Y. (2010). Formulation and characterization of matrix and triple-layer matrix tablets for oral controlled drug delivery. International Journal of Pharmacy 1075 and Pharmaceutical Sciences, 2(3), 137-143.

Tobyn, M. J., Staniforth, J. N., Baichwal, A. R., \& McCall, T. W. (1996). Prediction of physical properties of a novel polysaccharide controlled release system. I. International Journal of Pharmaceutics, 128, 113-122.

Turcotte, R. F., Lavis, L. D., \& Raines, R. T. (2009). Onconase cytotoxicity relies on the

1080 distribution of its positive charge. The FEBS journal, 276(14), 3846-3857.

Wang, F., Wang, Y. J., \& Sun, Z. (2002). Conformational role of xanthan in its interaction with locust bean gum. Journal of Food Science, 67(7), 2609-2614.

Wang, J., Yang, T., Tian, J., Liu, W., Jing, F., Yao, J., Zhang, J., \& Lei, Z. (2014). Optimization of reaction conditions by RSM and structure characterization of sulfated locust bean gum.

1085 Carbohydrate Polymers, 114, 375-383.

Yuan, H., Zhang, W., Li, X., Lu, X., Li, N., Gao, X., \& Song, J. (2005). Preparation and in vitro antioxidant activity of $\mathrm{k}$-carrageenan oligosaccharides and their oversulfated, acetylated, and phosphorylated derivatives. Carbohydrate Research, 340, 685-692.

Zhao, F., Zhao, Y., Liu, Y., Chang, X., Chen, C., \& Zhao, Y. (2011). Cellular uptake, intracellular

1090 trafficking, and cytotoxicity of nanomaterials. Small, $7(10), 1322-1337$. 


\section{Supplementary material}
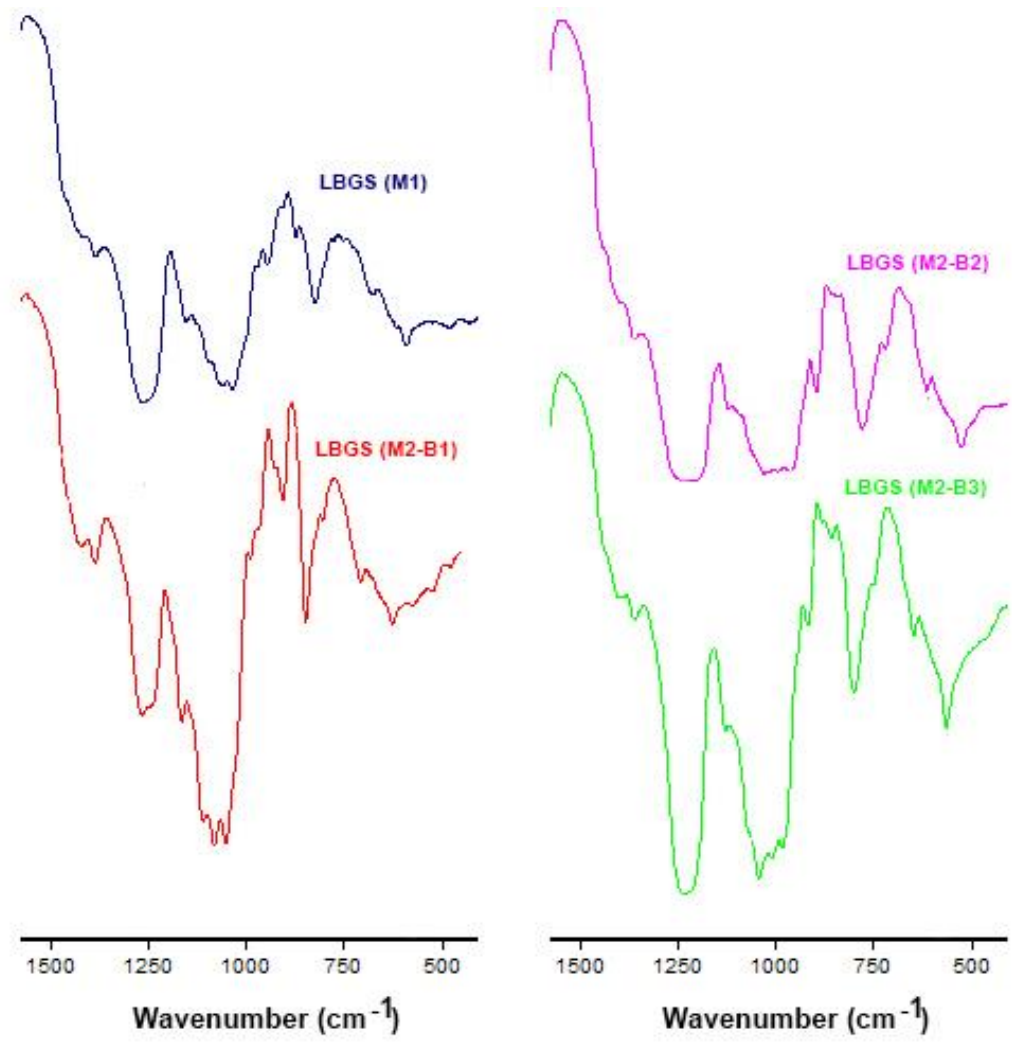

Figure S1 - FTIR spectra of Locust Bean Gum sulfate derivatives (LBGS) obtained in method 1 (M1) and method 2 (M2). B1, B2 and B3 refer to batch 1, 2 and 3, respectively. 


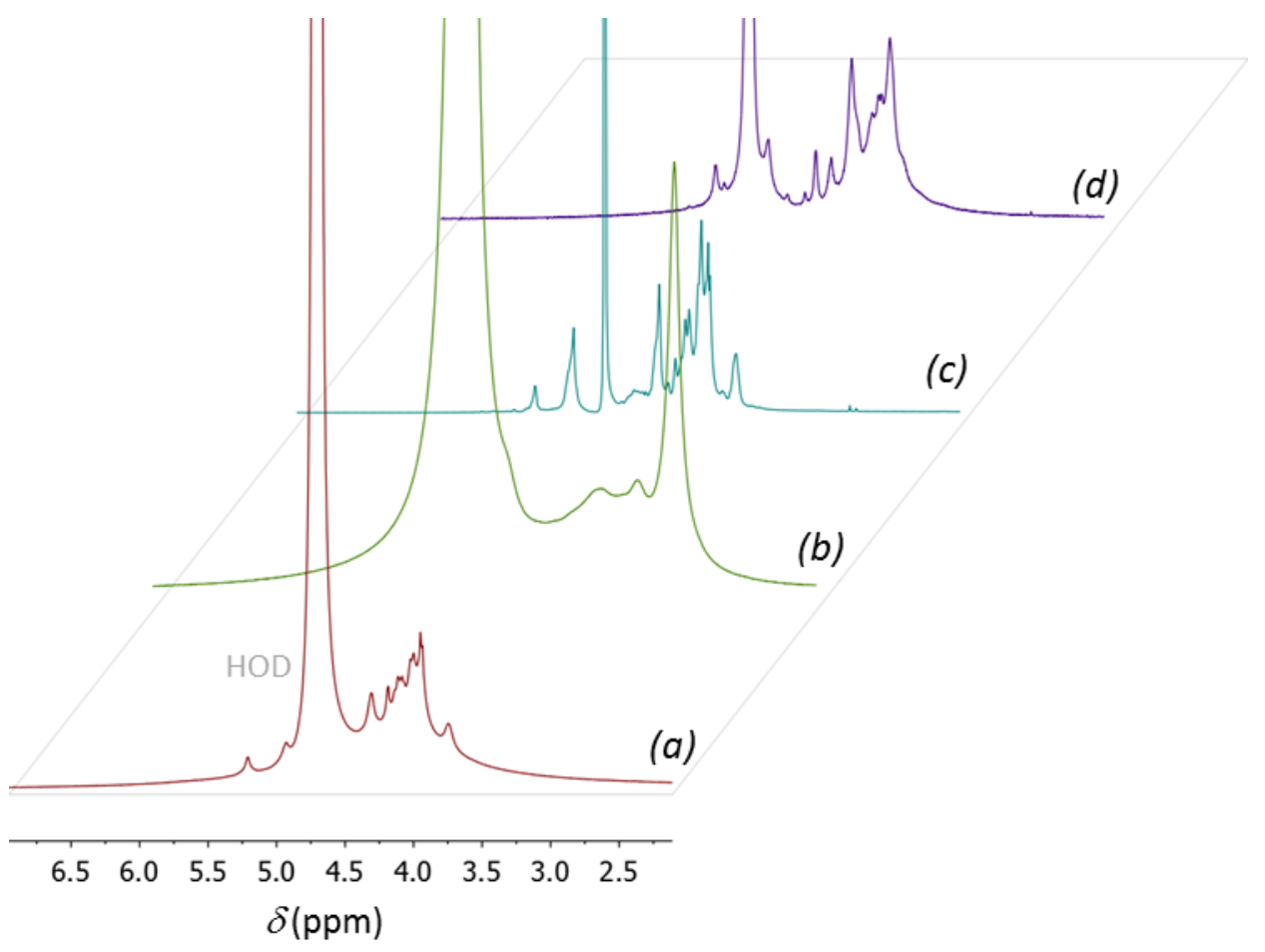

e)
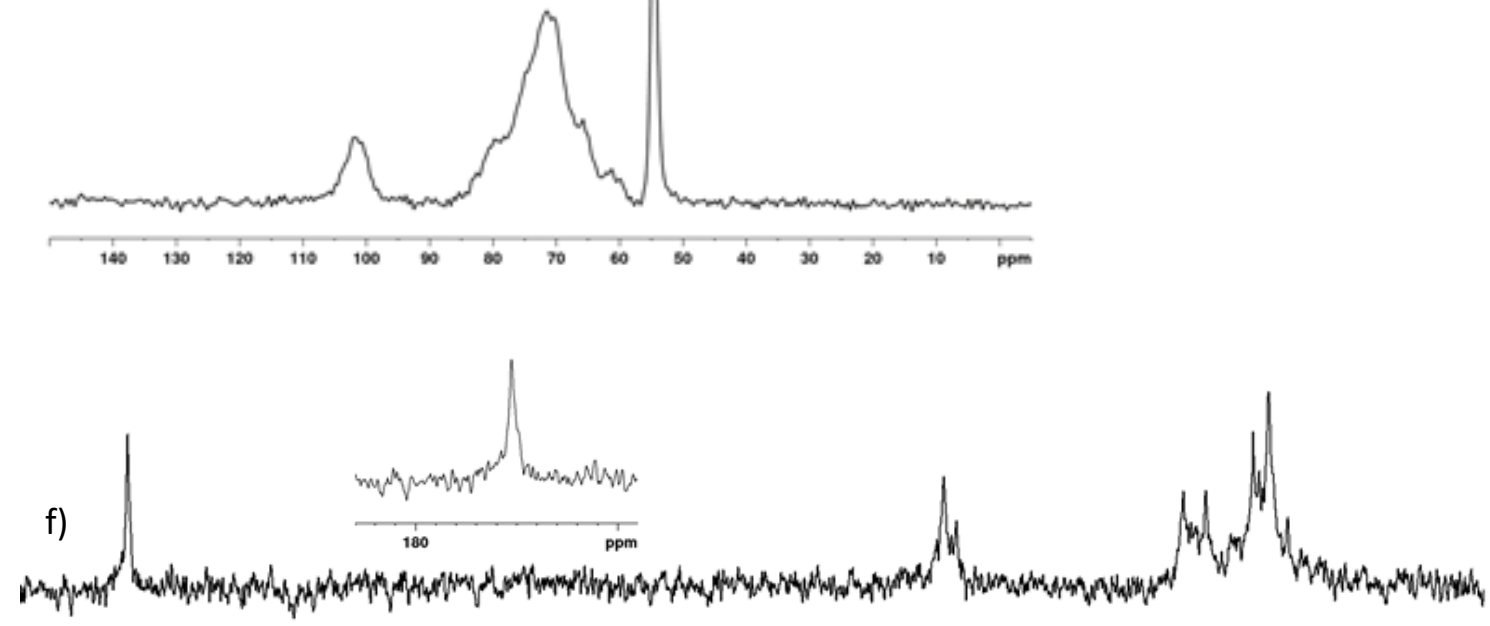

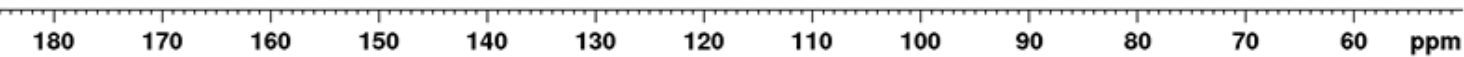

Figure S2 - ${ }^{1}$ H NMR spectra of (a) LBG, (b) LBGS-M2-B1, (c) LBGC, and (d) LBGA; (e) ${ }^{13} \mathrm{C}$ CPMAS spectrum of LBGA; (f) ${ }^{13} \mathrm{C}$ NMR spectrum of LBGC; the big singlet centered at $4.7 \mathrm{ppm}$ in the ${ }^{1} \mathrm{H}$ spectra is due to HOD (identified in grey). 


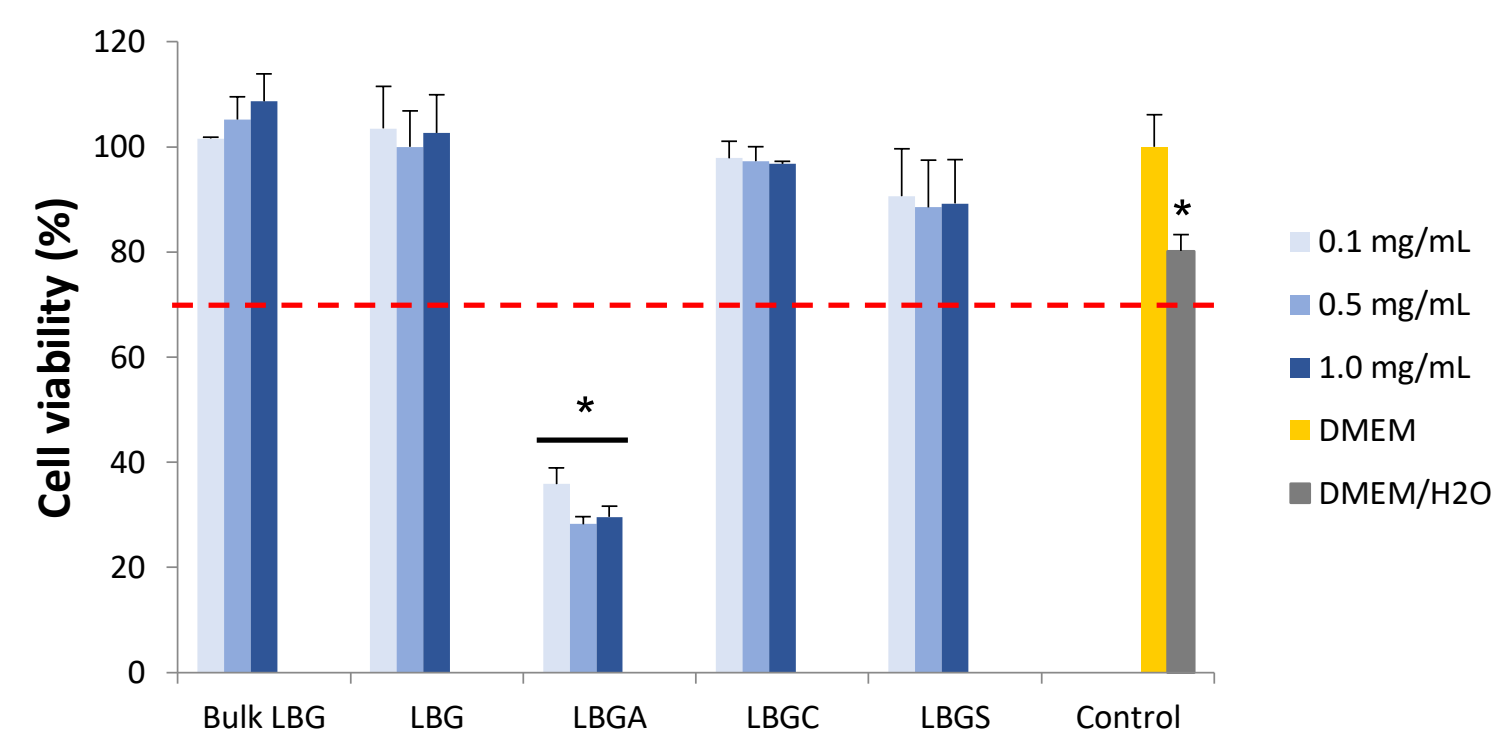

Figure S3 - Caco-2 cell viability measured by the MTT assay after $3 \mathrm{~h}$ exposure to increasing concentrations of bulk Locust Bean Gum, purified Locust Bean Gum (LBG) and its ammonium (LBGA), carboxylate (LBGC) and sulfate (LBGS) derivatives. Data represent mean \pm SEM ( $\mathrm{n} \geq 3$, six replicates per experiment at each concentration). Dashed line indicates $70 \%$. ${ }^{*} P<0.05$ compared with DMEM.

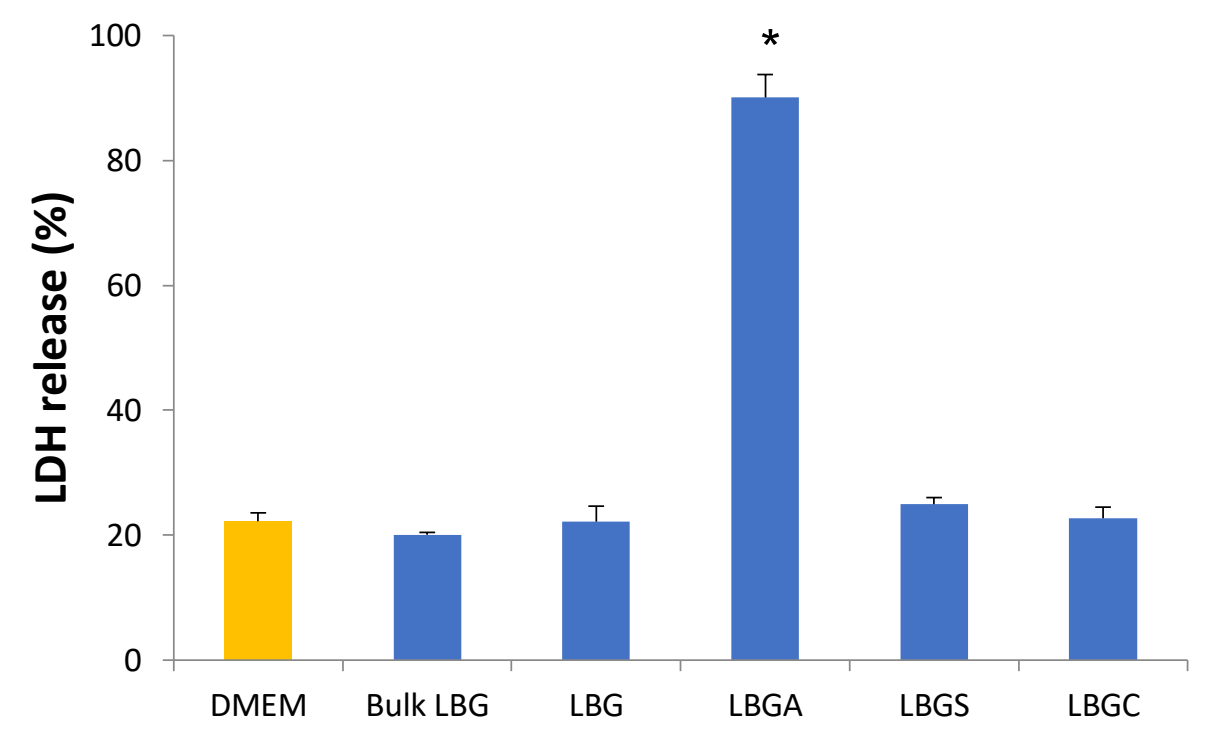

Figure S4 - Caco- 2 cell viability measured by the LDH release assay after $24 \mathrm{~h}$ exposure to $1 \mathrm{mg} / \mathrm{mL}$ solutions of bulk Locust Bean Gum, purified Locust Bean Gum (LBG) and its ammonium (LBGA), carboxylate (LBGC) and sulfate (LBGS) derivatives. Data represent mean \pm SEM ( $\mathrm{n} \geq 3$, three replicates per experiment). ${ }^{*} P<$ 0.05 compared with DMEM. 


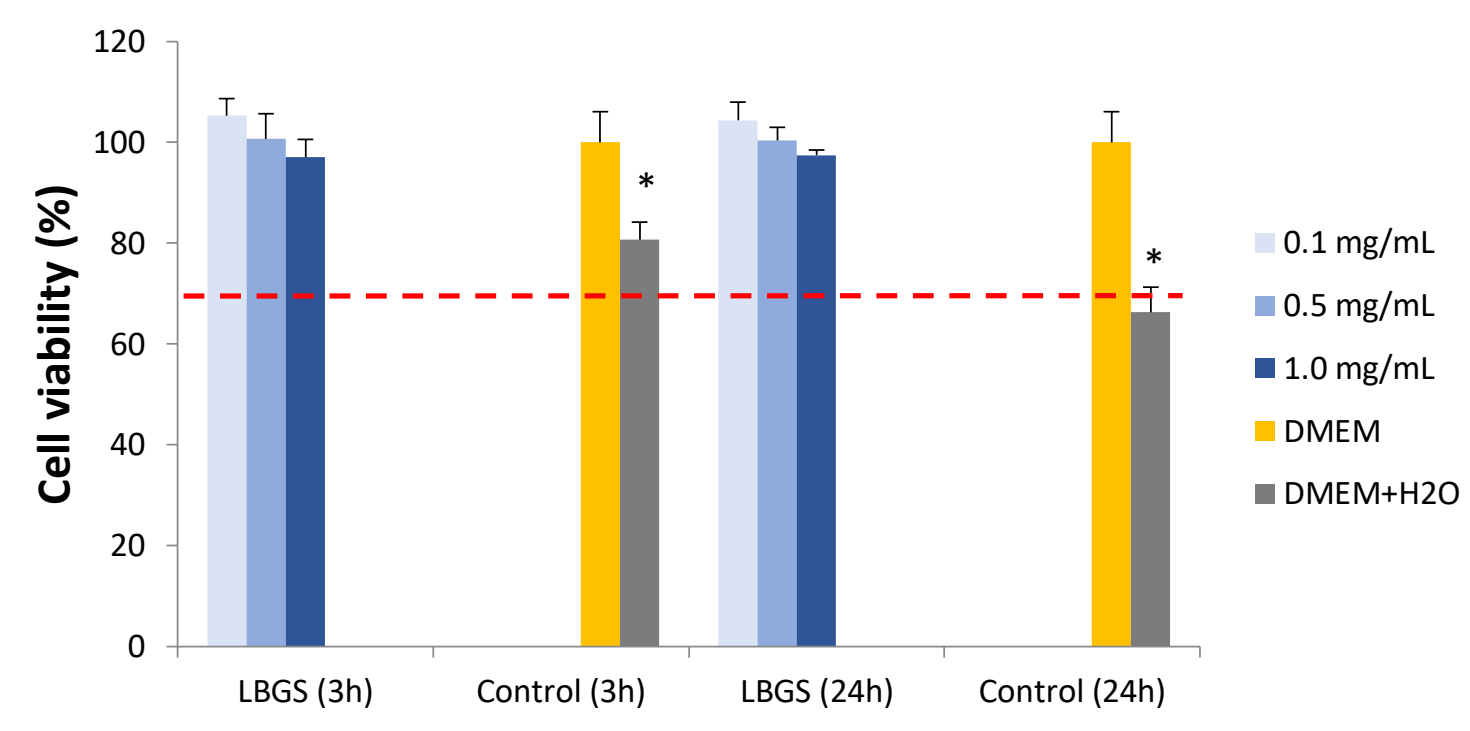

Figure S5 - A549 cell viability measured by the MTT assay after $3 \mathrm{~h}$ and $24 \mathrm{~h}$ exposure to increasing concentrations of sulfate locust bean gum (LBGS) derivative. Data represent mean $\pm \operatorname{SEM}(\mathrm{n} \geq 3$, six replicates per experiment at each concentration). Dashed line indicates $70 \%$. $* P<0.05$ compared with respective control (DMEM).

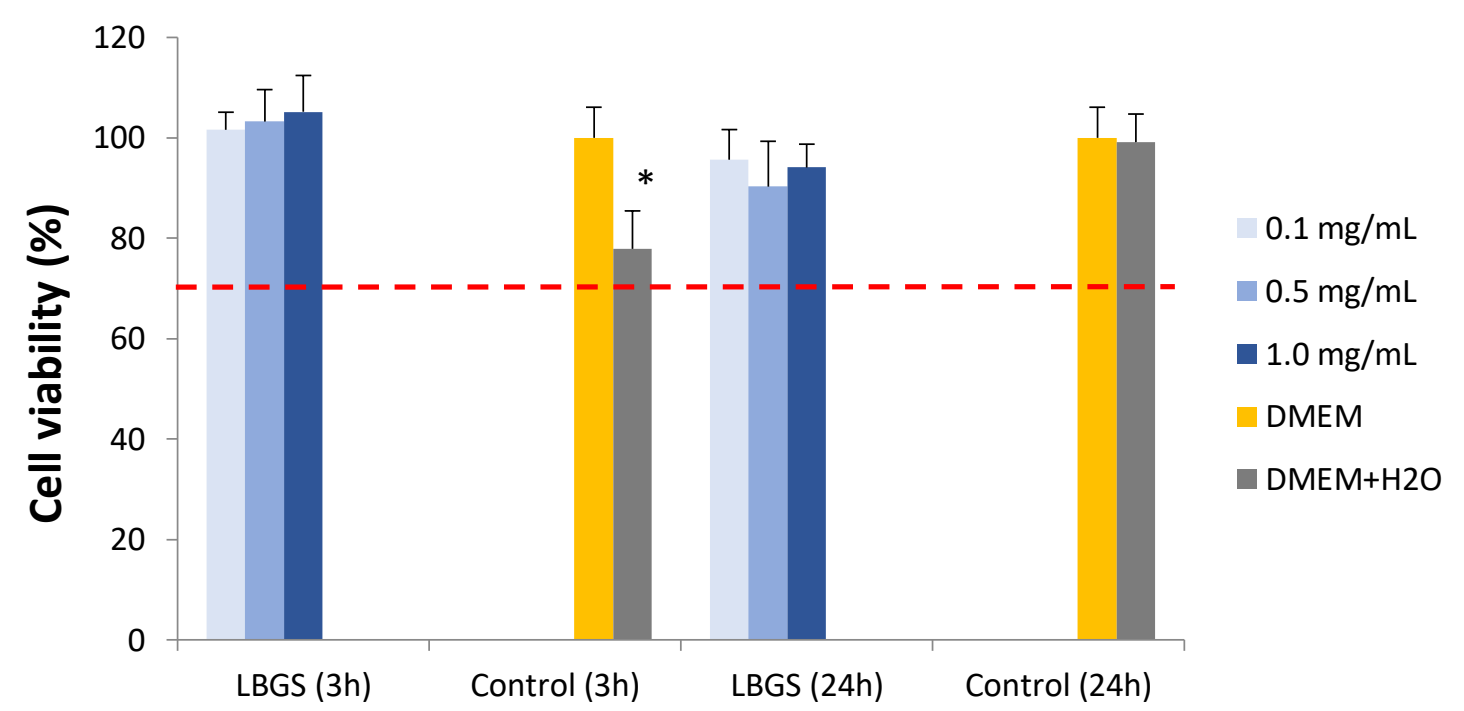

Figure S6 - Calu-3 cell viability measured by the MTT assay after $3 \mathrm{~h}$ and $24 \mathrm{~h}$ exposure to increasing concentrations of sulfate locust bean gum (LBGS) derivative. Data represent mean $\pm \operatorname{SEM}(\mathrm{n} \geq 3$, six replicates per experiment at each concentration). Dashed line indicates $70 \%$. $* P<0.05$ compared with respective control (DMEM). 


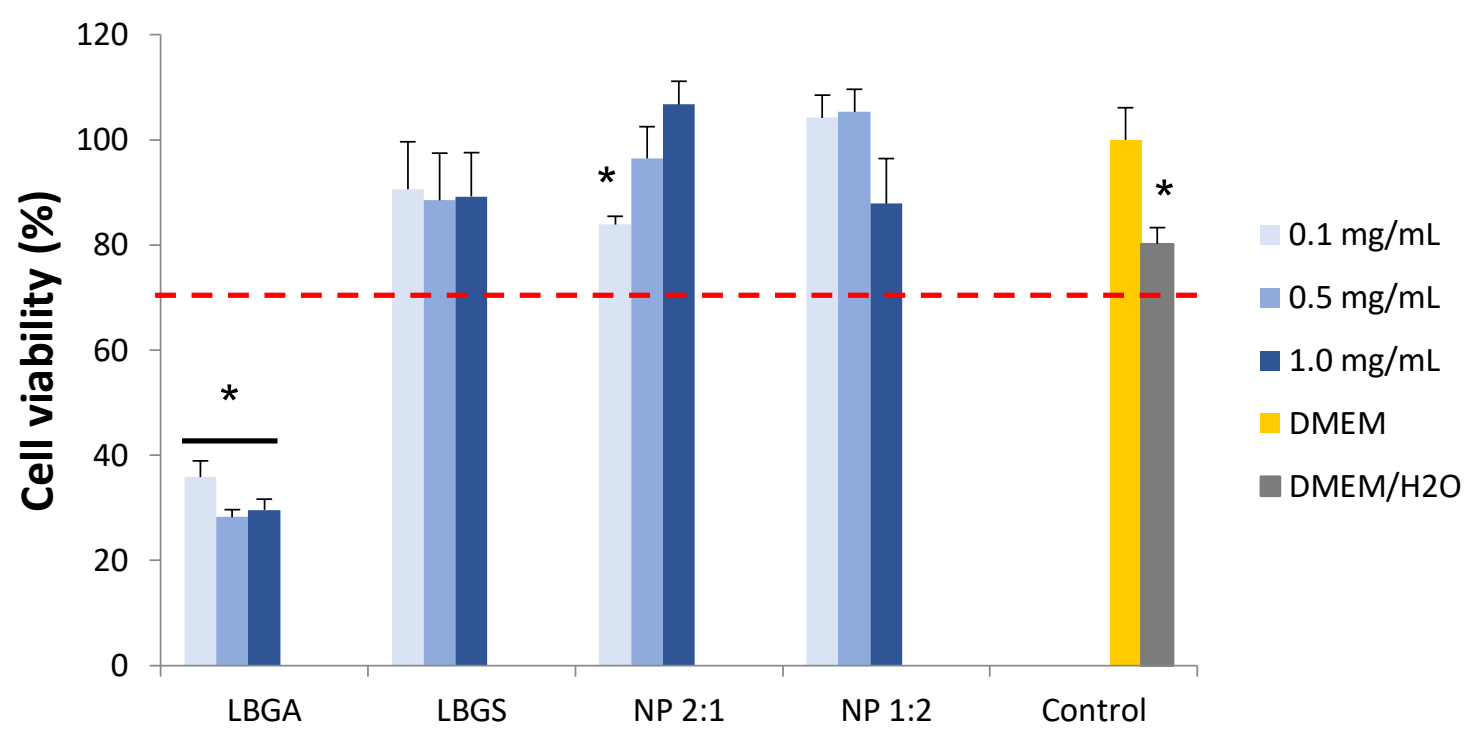

Figure S7 - Caco-2 cell viability measured by the MTT assay after $3 \mathrm{~h}$ exposure to increasing concentrations of ammonium Locust Bean Gum (LBGA) derivative, sulfate Locust Bean Gum (LBGS) derivative and LBGA/LBGS nanoparticles (NP). Data represent mean $\pm \operatorname{SEM}(\mathrm{n} \geq 3$, six replicates per experiment at each concentration). Dashed line indicates $70 \%$. $* P<0.05$ compared with DMEM.

Table S1 - Elemental analysis data from the sulfate (LBGS), carboxylate (LBGC) and ammonium (LBGA) derivatives of locust bean gum (LBG).

\begin{tabular}{c|cccccc}
\hline \multirow{2}{*}{$\begin{array}{c}\text { Element } \\
(\%)\end{array}$} & \multicolumn{6}{|c}{ Polymer } \\
\cline { 2 - 7 } & LBGS (M1)* & $\begin{array}{c}\text { LBGS (M2- } \\
\text { B1)* }\end{array}$ & $\begin{array}{c}\text { LBGS (M2- } \\
\text { B2)* }\end{array}$ & $\begin{array}{c}\text { LBGS (M2- } \\
\text { B3)* }\end{array}$ & LBGC & LBGA \\
\hline $\mathbf{N}$ & --- & --- & --- & --- & -- & 3.84 \\
$\mathbf{C}$ & 25.55 & 35.06 & 23.94 & 28.42 & 37.39 & 43.39 \\
$\mathbf{S}$ & 7.77 & 3.50 & 9.78 & 7.41 & --- & -- \\
$\mathbf{O}$ & --- & --- & --- & --- & 48.96 & -- \\
\hline
\end{tabular}

${ }^{*} \mathrm{~B} 1, \mathrm{~B} 2$ and B3 refer to LBGS derivatives from batches 1, 2 and 3, respectively; M1 and M2 refer to LBGS derivatives synthesized with methods 1 and 2 , respectively 
Table S2 - GPC analysis of purified Locust Bean Gum (LBG), and its ammonium (LBGA), carboxylate (LBGC) and sulfate (LBGS-M1) derivatives.

\begin{tabular}{ccccc}
\hline Polymer & $\boldsymbol{M}_{\mathrm{n}}(\mathrm{Da})$ & $\boldsymbol{M}_{\mathrm{w}}(\mathrm{Da})$ & Pdl & $\boldsymbol{R}_{\mathrm{g}}(\mathrm{nm})$ \\
\hline LBG & 327300 & 589100 & 1.80 & 71.61 \\
LBGA & 500600 & 871000 & 1.74 & 86.05 \\
LBGC & 73790 & 119500 & 1.62 & 28.19 \\
LBGS-M1 & 21380 & 26510 & 1.24 & 14.21 \\
\hline
\end{tabular}

$M_{\mathrm{n}}$ : number average molecular weight; $M_{\mathrm{w}}$ : weight average molecular weight; Pdl: polydispersity index; $R_{g}$ : radius of gyration 